\title{
Confocal Microscopy Reveals Coordinated Calcium Fluctuations and Oscillations in Synaptic Boutons
}

\author{
Naomi Melamed, Paul Johannes Helm, ${ }^{a}$ and Rami Rahamimoff \\ Department of Physiology, Hebrew University-Hadassah Medical School, Jerusalem 91010, Israel
}

\begin{abstract}
Calcium ions are one of the main factors regulating quantal transmitter release and thus synaptic transmission in the nervous system. Using confocal microscopy, fluorescent imaging with the calcium indicator Rhod-2, and time series analysis, we show that the levels of calcium ions inside single synaptic boutons of the lizard neuromuscular junction are not constant at rest, but undergo coordinated fluctuations in the space domain, which cover a large fraction of the synaptic bouton. Furthermore, oscillations in intracellular calcium were frequently observed in the time domain.

Control experiments showed no coordinated fluctuations or oscillations at locations outside the synaptic boutons. Edge detection analysis showed that the coordinated fluctuations and oscillations were not due to movement artifacts. No coordinated fluctuations and oscillations were seen when similar measurements and analyses were performed on artificial fluorescent beads.

A variance analysis was performed on artificial fluorescent beads and on synaptic boutons. The variance of the fluorescent signal at the synaptic boutons was larger than the variance in artificial beads with the same mean fluorescence. This extra variance was greatly reduced when the extracellular calcium concentration was decreased from 2.0 $\mathrm{mM}$ to $0.4 \mathrm{~mm}$.
\end{abstract}

We conclude that the coordinated fluctuations and oscillations in the calcium-induced fluorescence at the synaptic boutons are genuine biological phenomena and may be of significance in the regulation of transmitter release.

[Key words: calcium, confocal microscopy, oscillation, synaptic boutons, neuromuscular junction, synaptic transmission, lizard, Rhod-2, time series analysis]

The two main processes that determine and regulate synaptic transmission in the nervous system are the release of neurotransmitter from the presynaptic nerve endings and its detection by the postsynaptic cell. The release of transmitter is critically dependent on calcium ions both at peripheral (Dodge and $\mathrm{Ra}$ -

\footnotetext{
Received May 12, 1992; revised July 29, 1992; accepted Aug. 6, 1992.

We thank Drs. Vered Aviv, Ellen F. Barrett, Gavriel David, Simona Ginsburg, Igor Kaiserman, Halina Meiri, John Moore, Eli Nelken, Hannah Rahamimoff Bert Sakmann, and Nilly Yakir for very helpful discussions and comments during the preparation of the manuscript, Dr. Gavriel David for showing us the dissection of the lizard neuromuscular preparation, and Mrs. Marsha Rapp for secretarial assistance. The work and the purchase of equipment were supported by grants from the MDA, The Israeli Academy of Sciences and Humanities, U.S.-Israel BSF, GIF, and CTR.

Correspondence should be addressed to Rami Rahamimoff, Department of Physiology, Hebrew University-Hadassah Medical School, P.O. Box 1172, Jerusalem 91010 , Israel.

aPermanent address: Department of Cell Physiology, Max Planck Institute for Medical Research, D-6900 Heidelberg 1, Germany.

Copyright (C) 1993 Society for Neuroscience $0270-6474 / 93 / 130632-18 \$ 05.00 / 0$
}

hamimoff, 1967; Katz, 1969; Augustine et al., 1987) and at central synapses. In spite of its importance, we still do not know whether the free $\mathrm{Ca}^{2+}$ concentration in the nerve terminals in general, and in the synaptic boutons in particular, is constant at rest, or whether it fluctuates. The main difficulty in examining this question by conventional imaging techniques stems from the fact that the nerve terminals are usually covered by Schwann cells or glial cells, and one cannot be certain whether the optical signals originate from the nerve terminals or from the covering cells. This problem became even more evident recently with the finding that under certain experimental conditions, calcium waves could be detected in cultured astrocytes (Cornell-Bell et al., 1990; Jensen and Chiu, 1990). The uncertainty regarding the origin of calcium signals in a multicellular tissue can be overcome by confocal laser scanning microscopy, which possesses excellent lateral and axial resolution and therefore enables one to produce "optical sections" through living cells (Sheppard and Wilson, 1978; White et al., 1987; Pawley, 1990). We explored this approach at the lizard neuromuscular junction, which has well-defined synaptic boutons and excellent optical properties (Willard, 1915; Proske and Vaughan, 1968; Walrond and Reese, 1985). The physiology of this neuromuscular junction has been previously studied in detail (Proske and Vaughan, 1968; Barrett et al., 1988; Lindgren et al., 1988; Lindgren and Moore, 1989, 1991; Morita and Barrett, 1989, 1990).

Previously, confocal microscopy was used to study other aspects of the neuromuscular synapse (Lichtman et al., 1989; Robitaille et al., 1990; Melamed and Rahamimoff, 1991). In confocal microscopy, typically serial sections are made through the preparation and thereafter they are used to reconstruct a threedimensional object. In most of this work, we made a different usage of the confocal microscope. After making serial sections through a neuromuscular junction stained with a calcium probe Rhod-2 (Minta et al., 1989b), we chose a section that was well inside the synaptic bouton and scanned it repeatedly. Since both the covering Schwann cells and the muscle underneath the synaptic bouton lay outside the chosen confocal plane, their contribution to the light signals was negligible. Therefore, we were able to follow the changes that occur at a single plane inside the synaptic bouton. Using confocal microscopy in this way, we found that the calcium-induced Rhod-2 fluorescence is not stationary in time and space in the resting synaptic bouton, but shows coordinated fluctuations and oscillations.

A preliminary account of this work appeared in abstract form (Melamed et al., 1992).

\section{Materials and Methods}

Preparation and fluorescent staining. All experiments were performed on the isolated cerato-mandibularis nerve muscle preparation of the 

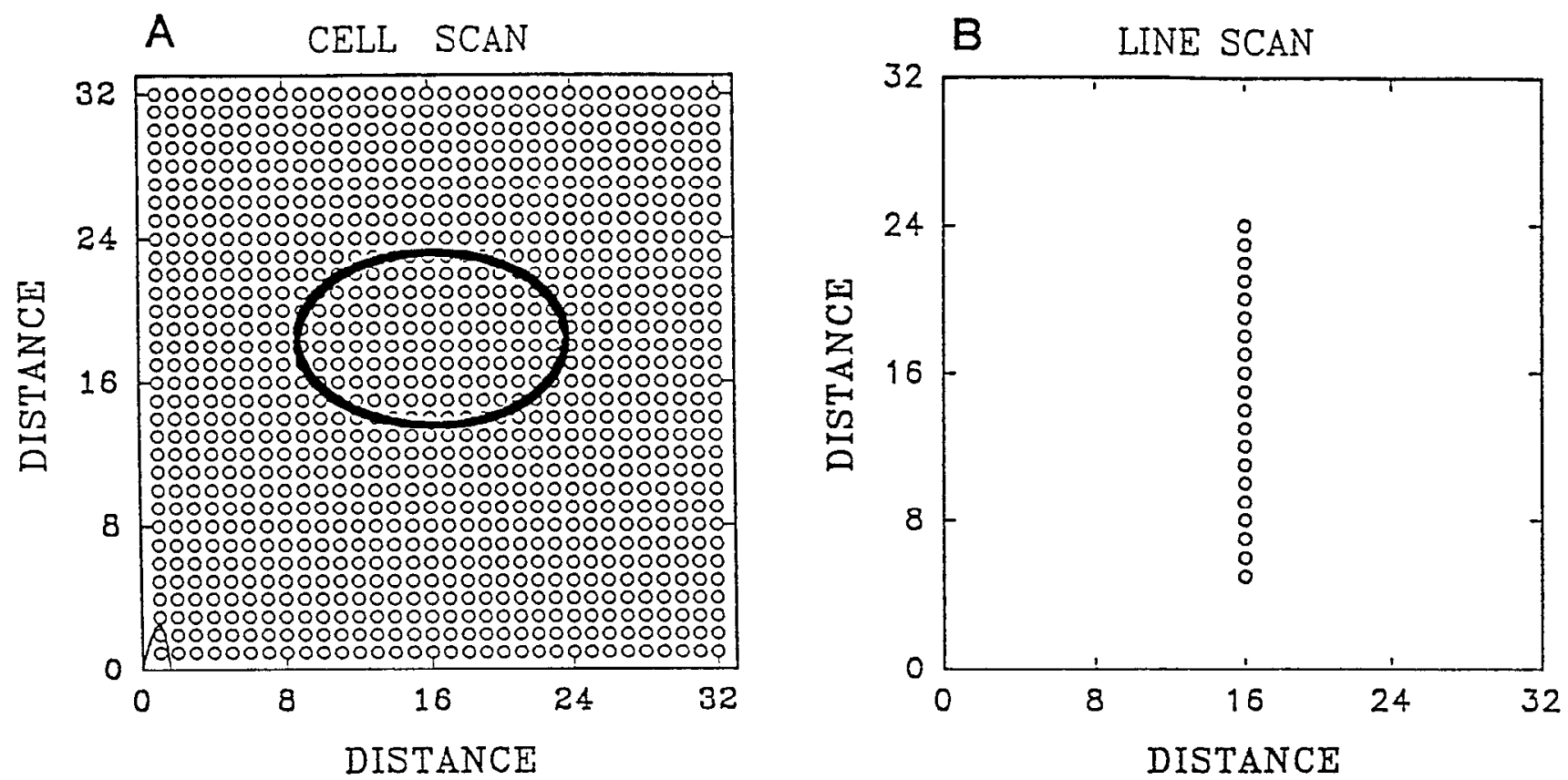

Figure 1. A diagrammatic representation of the two modes of confocal scanning used. $A$, Image scan. Each point represents a pixel. Usually the pixels were at $0.5 \mu \mathrm{m}$ distance. $B$, Line scan.

lizard Anolis carolinensis. The preparation was perfused during expcriments with normal lizard Ringer's containing (in $\mathrm{mM}$ ) $157 \mathrm{NaCl}, 4$ $\mathrm{KCl}, 2 \mathrm{CaCl}_{2}, 2 \mathrm{MgCl}_{2}, 5$ glucose, and 5 HEPES, pH 7.2-7.4. In the low-calcium Ringer's solution, [Ca] was lowered to $0.4 \mathrm{~mm}$ and $[\mathrm{Mg}$ ] was increased to $3.6 \mathrm{~mm}$ so that the total divalent ion concentration remained constant. Experiments were performed at room temperature $\left(20-24^{\circ} \mathrm{C}\right)$.

After dissection, the preparation was incubated in normal lizard Ringer's containing $4 \mu \mathrm{M}$ of the calcium indicator Rhod-2/AM (Molecular Probes, Eugene, OR) for $60 \mathrm{~min}$. After the staining, the preparation was washed extensively with normal lizard Ringer's solution.

The loading of the boutons with the dye was done by using the AM form of the dye (Minta et al., 1989a,b). Between the two calcium indicators (Fluo-3 and Rhod-2), Rhod-2 gave a better staining and was therefore used throughout this set of cxperiments. Rhod-2 has a number of advantages and disadvantages. The main advantage is that it works consistently. Its main disadvantage is that there is no spectral shift upon calcium binding, and therefore it is not a "ratio" dye (Grynkiewicz et al., 1985). We could not use routinely a calcium ionophore (such as ionomycin) to calibrate our fluorescence signal, since addition of an ionophore produced not only an increase in the optical signal, but also a mechanical displacement of the muscle, with a resulting loss of the scanning coordinates. Hence, all the data in this article are presented as fluorescence intensity and are not translated into absolute values of intracellular [Ca]. Control experiments were performed on fluorescent beads with a diameter of approximately $30 \mu \mathrm{m}$, obtained from Meridian Instruments, Inc. (Okemos, MI).

Confocal laser scanning microscopy. The experiments described in this article were performed on a confocal lascr scanning microscopc (CLSM) (Carlsson and Åslund, 1987), Sarastro Phoibos 1000 (Molecular Dynamics, Sunnyvale, CA). The scanner was mounted on a Zeiss Universal Microscope fitted with a $40 \times 0.75 \mathrm{NA}$ water immersion objective. Optical excitation was done by the $514 \mathrm{~nm}$ line of the argon ion laser. The emitted fluorescent light passed through a $530 \mathrm{~nm}$ (OG 530) long-pass filter before it reached the photomultiplier. The neutral density filter (NDF) was $100 \%$, unless stated otherwise in the figure caption.

The horizontal scanning in confocal laser scanning microscopy is done either by a stationary laser beam and a moving specimen stage or with a stationary specimen and a moving beam. In the Sarastro CLSM the latter procedure is used. Horizontal beam movements were achieved using two mirrors. One was a galvanometrically driven fast mirror (galvo-mirror), which swept the laser beam every $20 \mathrm{msec}(50 \mathrm{sweeps} / \mathrm{sec})$ along a straight line in the planc of focus. The line consistcd of 64-1024 pixels (pixel $\equiv$ picture element). The second was a slower step motor driven mirror moved by one step, every $20 \mathrm{msec}$. Thus, one line after the other was scanned. The time interval needed to scan two adjacent pixels thus depended on the direction. In the direction of the step motordriven mirror, the time interval between adjacent pixels was exactly 20 msec. In the direction of the galvo-mirror, the time interval was much shorter, and it depended on the number of pixels scanned. The $20 \mathrm{msec}$ of the galvo-mirror sweep consisted of $15 \mathrm{msec}$ of data collection and $5 \mathrm{msec}$ of flyback and resetting to the beginning of the line. The $15 \mathrm{msec}$ of data collection are divided by the number of pixels required. Therefore, the integration time of the photomultiplier depended on the number of pixels scanned; it is $14.6 \mu \mathrm{sec} /$ pixel for a 1024 pixel line and $234.3 \mu \mathrm{sec} / \mathrm{pixel}$ for a $64 \mathrm{pixel}$ line. Since photon statistics were much better for a smaller number of pixels, the typical line scan in our experiments was $64-128$ pixels.

The two scanning modes used in this article were the image scan (Fig. $1 A$ ) and the line scan (Fig. $1 B$ ). In the image scan, both mirrors were used, and the preparation was scanned at a relatively slow frequency. For example, a $128 \times 128$ pixel image was scanned in $2.56 \mathrm{sec}$, and afterward the data were transferred to the computer disk; the typical interval between scans was 3 or 4 sec. In the line scan mode, only the galvo-mirror was used, and therefore the time interval between sequential scans was $20 \mathrm{msec}$.

At the beginning of each experiment, the stained preparation was put on the confocal microscope and a series of sections in the three orthogonal directions was collected and used to select the optimal position of the preparation for image and line scans.

Data analysis. Data collection and analysis used programs supplied by Sarastro (Molecular Dynamics) and several commercially available programs (SIGMAPLOT, Jandel; and STATGRAPHICs, STSC).

The adaptation of the line scan program to our specific needs was done by one of us (P.J.H.) to include a three-dimensional display of the data and an interactive viewing of selected lines or pixels as a function of time.

A set of programs were written to analyze the fluorescent signals (R.R.). These programs consist of a correlation subroutine, which was incorporated into a number of different programs to perform autocorrelations, cross-correlations, and correlation maps of the neuromuscular junction, using an equal number of data points/lag. For autocorrelation, the correlation coefficients were calculated on the same array with an appropriate lag. The lag taken was the time interval between two successive scans in the line scan experiments. For cross-correlation maps, the correlation coefficients were calculated for each pair of pixels in the map. Programs were written to estimate the instrumental, methodological, and biological components in the variance of the fluorescent signals.

The size of the laser spot is approximately $1 \mu \mathrm{m}$. The typical dis- 
placement in most of the experiments was $0.5 \mu \mathrm{m}$. Hence, each location in the preparation was illuminated twice during the line scans. To check whether this double illumination had any appreciable effect on the estimated correlations, we compared the correlations between adjacent pixels along the line with correlations between the adjacent pixels in two separate lines, and did not observe any significant difference (see Fig. 4).

\section{Results}

\section{Calcium fluctuations in synaptic boutons: image scan} experiments

Figure 2 shows qualitatively spontaneous changes in Rhod-2 fluorescence in part of a neuromuscular junction; the intensity of the calcium signal is coded by pseudocolor. Figure $2 A$ illustrates a single image scan through the synaptic region. Signals coming from the boutons in the nerve terminals were much stronger than those from the background and from the muscle. Some of the boutons were better stained than others. For analysis, we chose boutons with fluorescence that did not reach saturation in any one of the scans. For pictorial presentation of the fluctuations, we selected the portion of the nerve terminal enclosed by the red square in Figure $2 A$ and magnified it digitally (there is no further resolution in such a magnification); 10 consecutive image scans (out of 335), all obtained at the same focal plane, are illustrated in Figure $2 B-K$. Fluorescence intensity clearly fluctuated over time, and the location of the maximal fluorescence varied from scan to scan. Figure $2 L$ illustrates the procedure used to estimate the boundaries of the synaptic boutons; all 335 scans of this experiment were summed to produce "saturation" of the pseudo color coding.

\section{Calcium fluctuations are coordinated in the space domain}

The observation of fluctuations in intracellular calcium fluorescence is not surprising, since almost all chemical and biological processes fluctuate on the microscopic level. We were interested, however, in examining whether these fluctuations were completely random in the space and time or whether they followed some inherent "order" (in the statistical sense). Figures 3 and 4 show, in two different ways, that the fluctuations in intracellular fluorescence were not random in the space domain, and that changes recorded at each individual pixel were well correlated with those in neighboring pixels. In Figure 3, each individual scan was transformed into a "calcium map" where the $\mathrm{x}$ - and $\mathrm{z}$-coordinates indicate the position in the confocal plane, and the $y$-coordinate shows the Rhod-2 fluorescence. All the calcium maps were averaged, and the average map recorded from the entire neuromuscular junction is shown in Figure $3 A$. Figure $3 B$ illustrates the average map of one single bouton, using a slightly different observation angle from that employed in Figure $3 A$. The average calcium map of this bouton was then subtracted from each individual scan, and difference maps were produced, in which the average fluorescence for the entire series was at the zero plane. All pixels that had a fluorescence above the average lie above the zero plane, and all pixels below the average lie below the zero plane. We expected that if the calciuminduced fluorescence in each location fluctuated randomly and independently of the neighboring pixels, then the distribution of pixels with above-average or below-average fluorescence would be random. The results of difference analysis for 2 out of the 145 scans obtained in this experiment are shown in Figure $3 C$ $F$. Figure $3 C$ shows the calcium map of one individual scan, and the difference between this calcium map and the average map in Figure $3 B$ is shown in Figure $3 D$. Figure $3, E$ and $F$, represents the results of another scan following the same procedure as described for Figure $3, C$ and $D$. It is clear that the fluorescence fluctuations from scan to scan at each individual location were not independent of the neighboring locations; quite frequently, a substantial fraction of the entire bouton was simultaneously either below the average or above the average value, suggesting the existence of some coordination among adjacent locations.

To test further for coordinated calcium fluctuations in the synaptic bouton, we performed correlation analysis of fluorescence changes in adjacent pixels in the same bouton. In Figure $4 A$, the solid circles show the avcrage fluorcscence (of 335 scans) of each of 41 pixels along a scanning line, and the open circles represent the correlation coefficient (multiplied by 100) between the fluorescence array recorded at pixel 26 and each of the other pixels. The data show strong positive correlation between the reference pixel (pixel 26) and the adjacent pixels, which decayed steeply near the edges of the bouton, indicating that the coordinated fluctuations in $[\mathrm{Ca}]_{\text {in }}$ covered a large fraction of the bouton. In other words, these strong positive correlations show that if a certain location at a given time is above the average, then there is a very good chance that the adjacent locations will also be above their respective average.

Since confocal microscope first scans one line and then moves to the next line (see Materials and Methods), the time interval in scanning adjacent locations is only tens or hundreds of microseconds along the line, but it is $20 \mathrm{msec}$ between lines. Thus, we performed the correlation analysis in both scanning dircctions: in Figure 4, $B$ and $C$, the correlation was performed between the same pixel used in Figure $4 A$ and all the pixels in the preceding line (Fig. $4 B$ ) and the following line (Fig. $4 C$ ). In both cases, a significant correlation was observed, indicating that the factor (or factors) responsible for the coordinated fluctuation in the space domain last more than $20 \mathrm{msec}$. The analysis was not dependent on the pixel chosen within the bouton. The same type of analysis was performed in four additional neuromuscular preparations, with similar results

\section{Calcium oscillations in the time domain: line scan experiments}

The conclusion from the image scan experiments illustrated in Figures $2-4$ is that the calcium-induced fluorescence inside the bouton shows substantial fluctuations that are coordinated in the space domain. To obtain a faster resolution in the time domain, we scanned a single line confocally at any desired depth.

Figure 2. Fluctuations in the fluorescence of the $\mathrm{Ca}^{2+}$ indicator Rhod-2 in confocal optical sections of single synaptic boutons of the lizard neuromuscular junction. $A$, Scan 101 out of 335 consecutive scans all done at the same focal plane of the lizard neuromuscular junction. $B-K$ Consecutive scans 101-110: the two boutons in the red square in $A$ were magnified (and interpolated to give a full display); the time interval between two consecutive scans is approximately 4 sec. Note variability in fluorescence among scans; compare, for example, the scans in $F$ and $J$. $L$, A quick look-through projection (the fluorescence levels, in each pixel, of all 335 scans were summed); the projection shows the estimated borders of a single bouton. The preparation was continuously perfused with lizard Ringer's solution. Output laser power, $11.0 \mathrm{~mW}$. Photomultiplier sensitivity (PMT), 825; NDF, $10 \%$. Scanning of $128 \times 128$ pixels. Each pixel displacement was $0.5 \mu \mathrm{m}$. Scale bar: $A, 10 \mu \mathrm{m} ; B-L, 3.75 \mu \mathrm{m}$. 

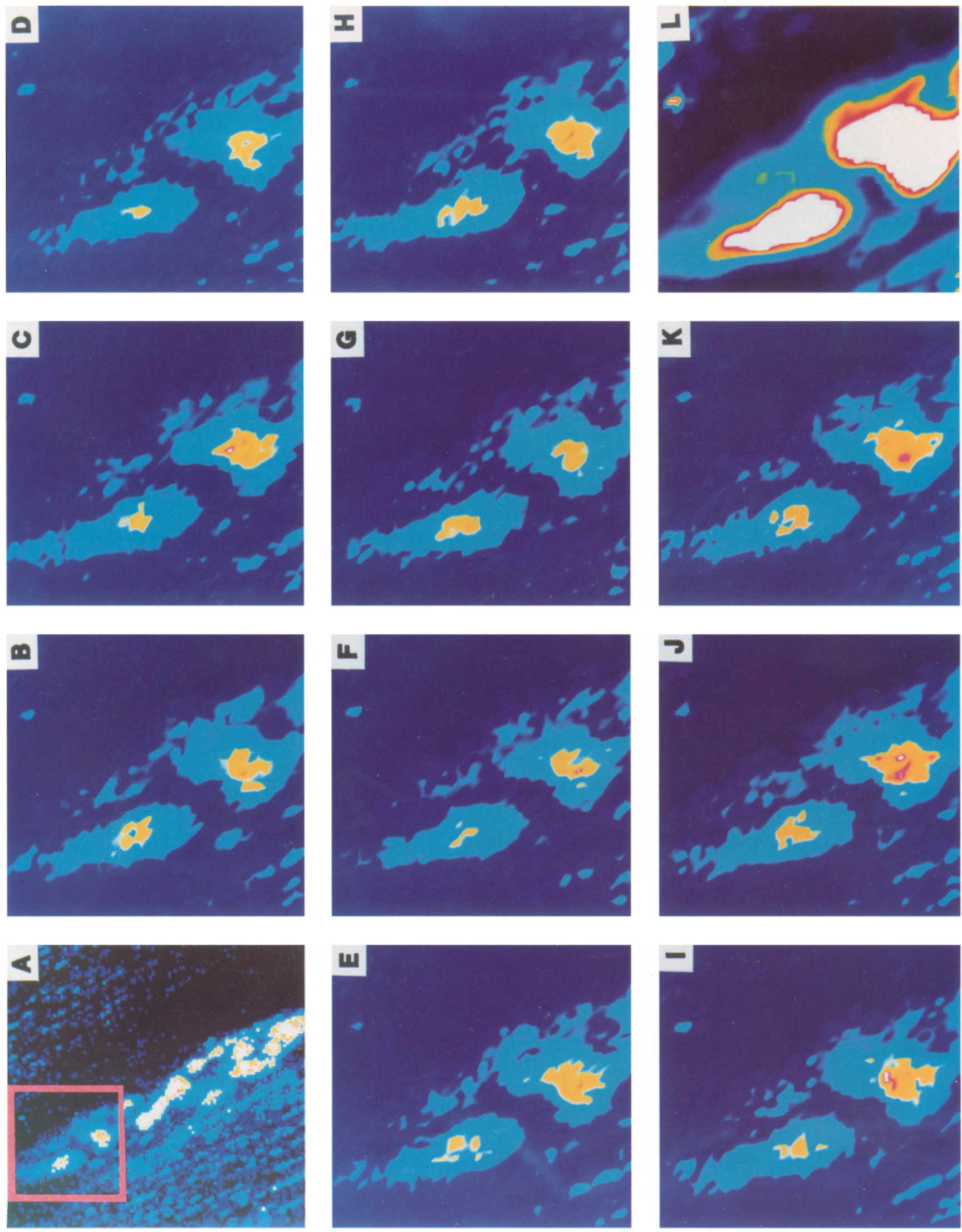


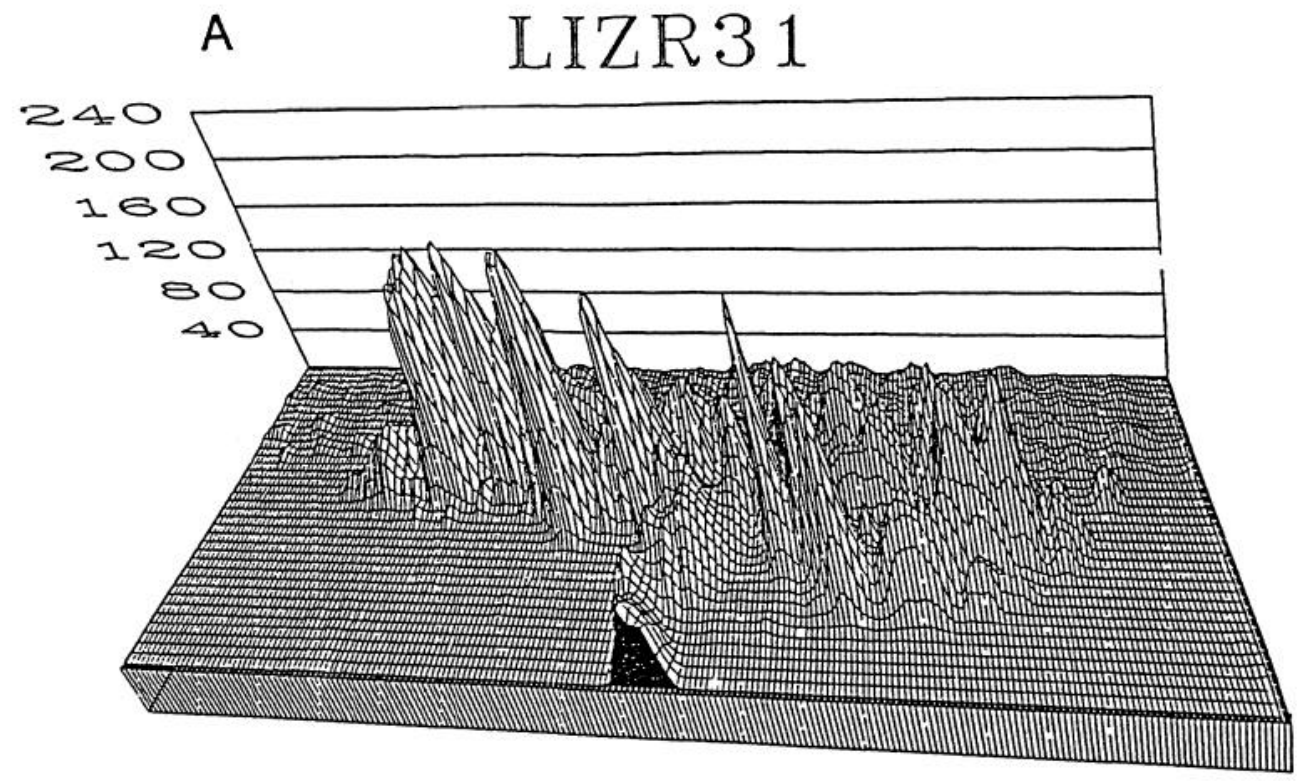

average scan

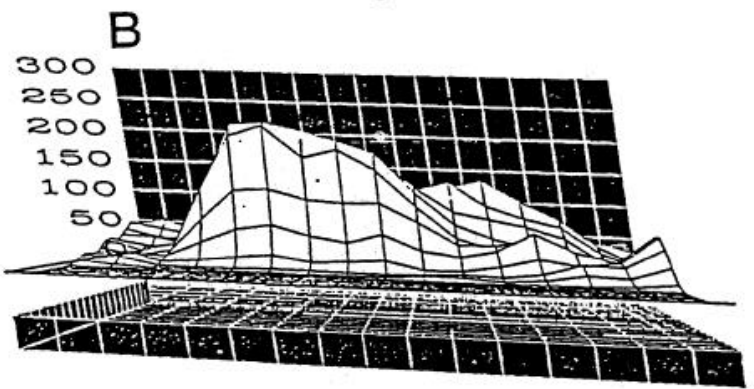

average scan

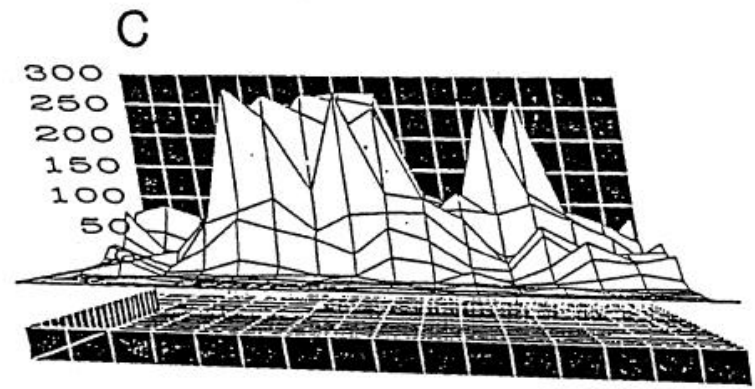

$\operatorname{scan} 30$

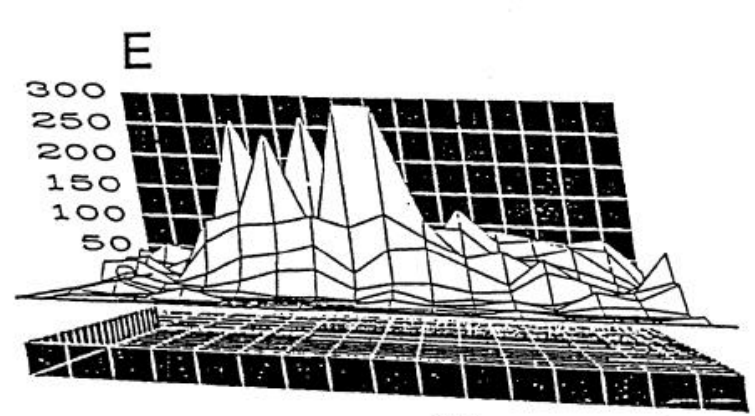

scan 109

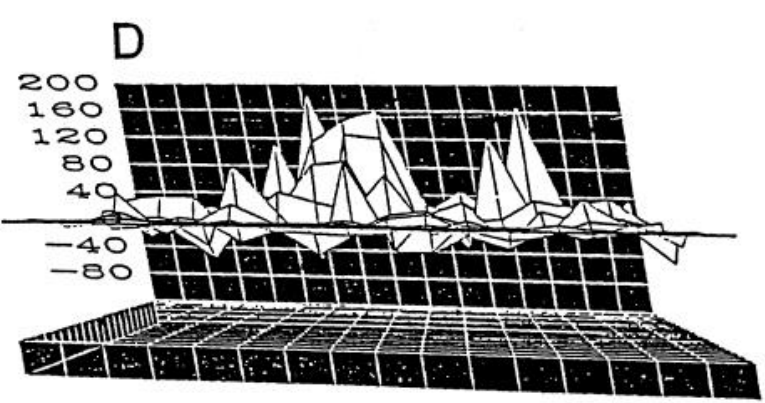

dill scan 30

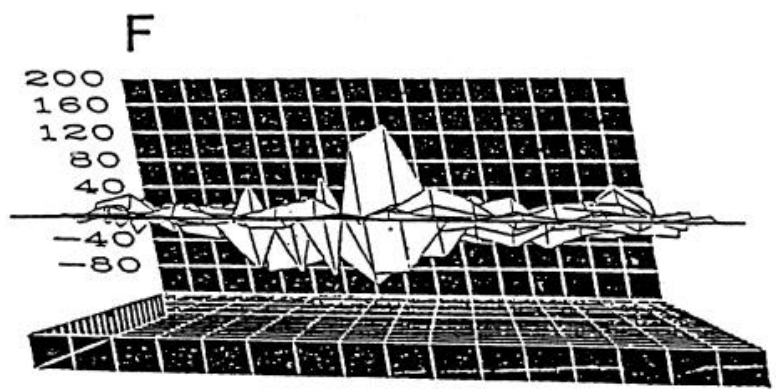

dill scan 109

Figure 3. Difference analysis of confocal calcium images of synaptic boutons. Fluorescence values obtained from the analog-to-digital conversion of the PMT output (gray scale 0-255) were transferred to Boeing 3D program; $x$ - and $z$-values represent locations, and the ordinate is Rhod-2 

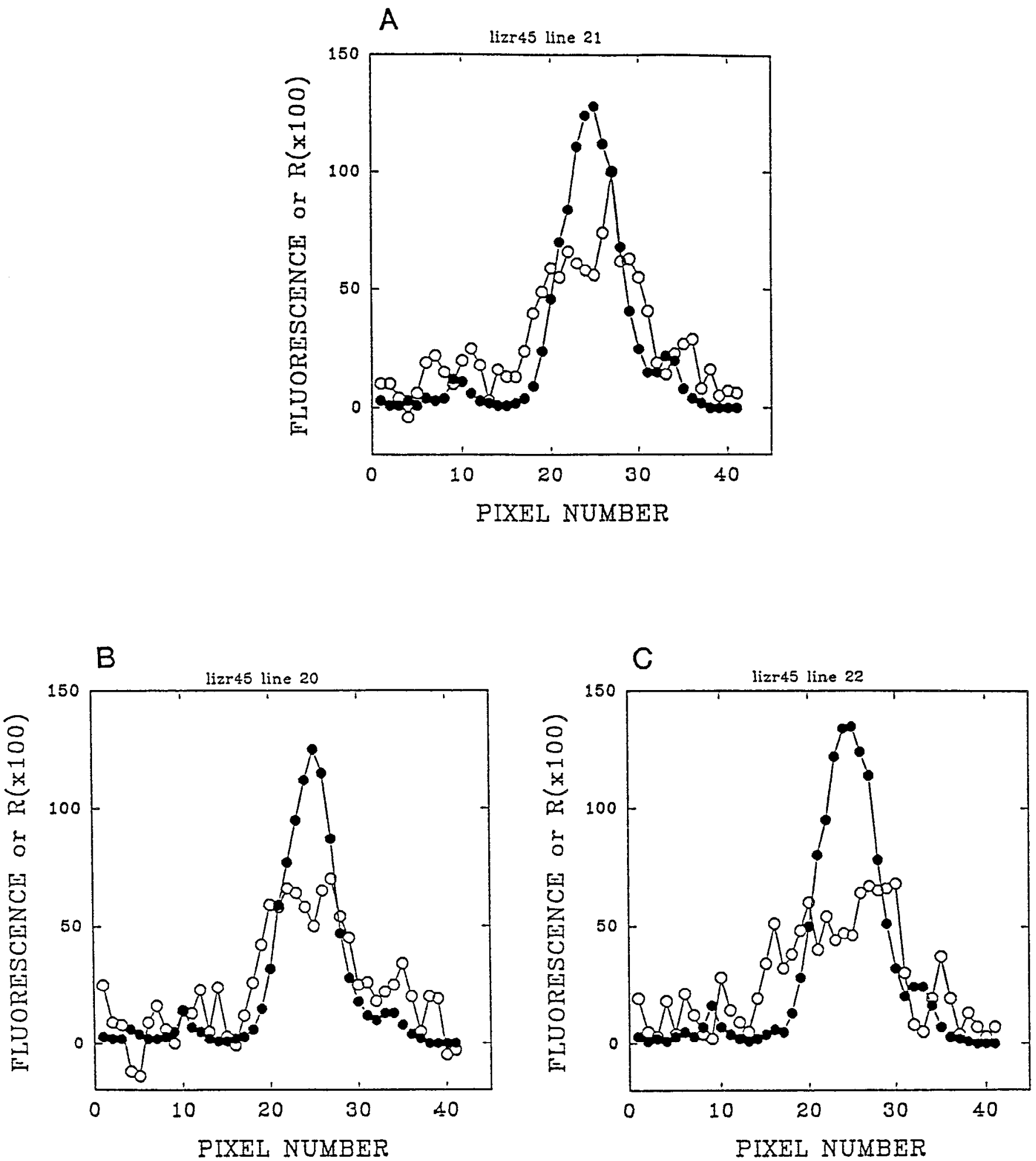

Figure 4. A correlation map of the calcium-induced Rhod-2 fluorescence in synaptic boutons. The analysis in $A-C$ was done on the data presented qualitatively in Figurc 2. A, Solid circles, are average fluorescence of all 335 scans. The image scans consisted of an area of $128 \times 128$ pixels at $0.5 \mu \mathrm{m}$ distance. Line 21 (out of 128$)$ is illustrated. Open circles are correlation coefficients $(\times 100)$. All correlation coefficients were calculated relative to pixel 26 . Note the significant positive correlation. $B$ and $C$, The same as $A$, but for the adjacent lines (20 and 22$)$, showing that the positive correlation is in both dimensions. Same experimental conditions as in Figure 2.

fluorescence. $A$, The average scan of the entire neuromuscular junction: 145 scans in the same focal plane. $B$, The averaged scan of the fourth bouton from the left in Figure $2 A$ was enlarged and served for the subsequent analysis. $C$, Single scan: a view from the zero level plane. $D$, Difference scan of the data in $C$, the averaged scan $(B)$ was subtracted from the single scan $(C)$, and the valucs are vicwed from the zero level plane. Note that most of the pixels are above the mean value. $E$ and $F$, The same as in $C$ and $D$, but for another single scan. Image size, $256 \times 256$ pixels; pixel size, $0.5 \mu \mathrm{m}$. PMT, 801 ; output laser power, $11.1 \mathrm{~mW}$. No perfusion. 

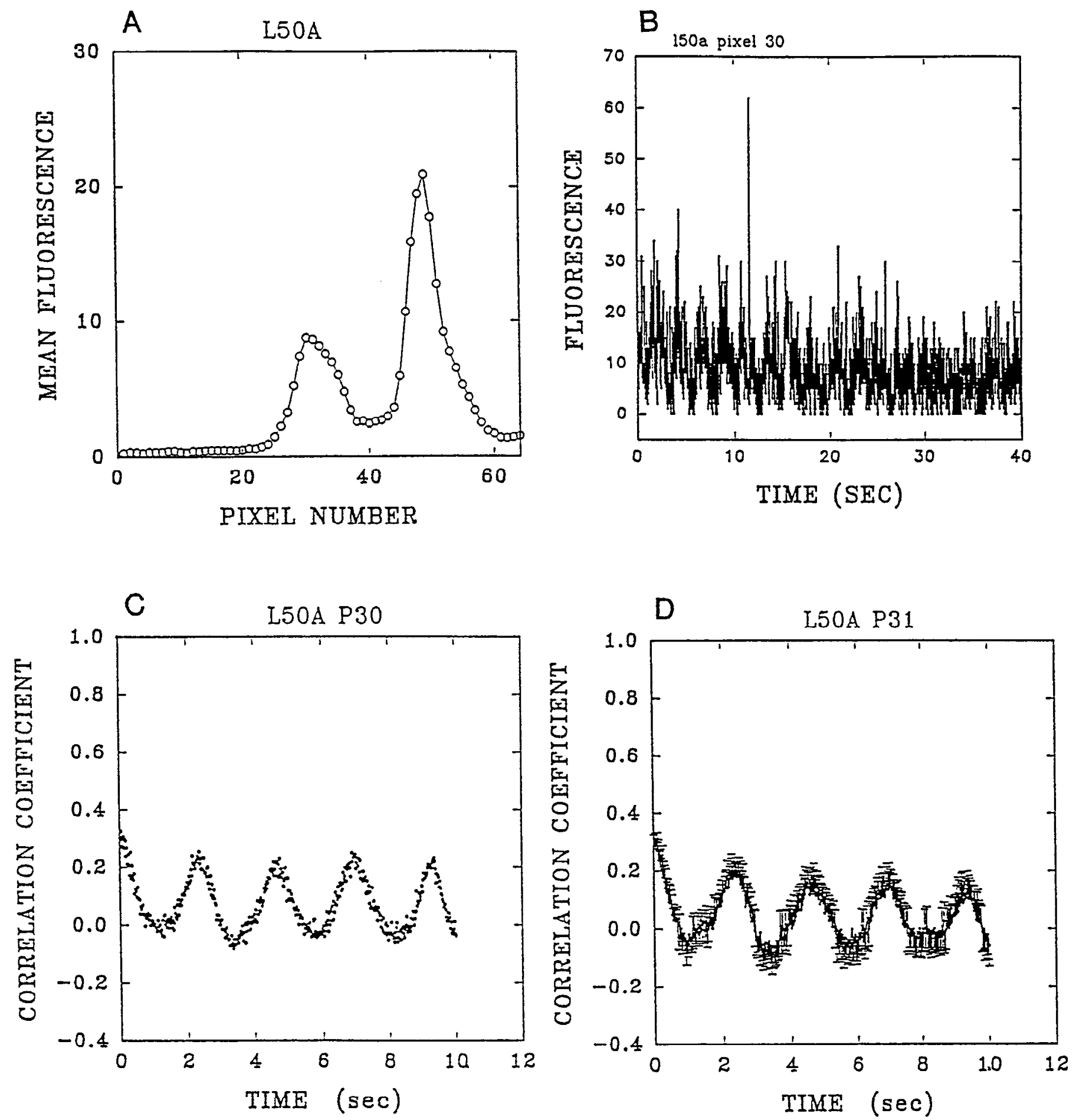

Figure 5. Calcium oscillations at a synaptic bouton of the lizard neuromuscular synapse. A line was placed across two synaptic boutons and 2000 line scans were collected at $50 \mathrm{~Hz}$. The line consisted of 64 pixels separated by $0.5 \mu \mathrm{m}$. The position of the line was determined by a preliminary series of image scans at various confocal planes. $A$, Average fluorescence of all 2000 scans plotted as a function of the pixel number. $B$, The 2000 consecutive data points of pixel 30 . To check for bleaching, a linear regression analysis was performed on the experimental data. The regression line follows the equation $y=-0.003 x+11.83$, where $y$ is the fluorescence and $x$ is the scan number $(25 \%$ loss of fluorescence in 1000 scans in this experiment). $C$, Autocorrelation of the data in $B$. Unitary lag of $20 \mathrm{msec}$ (one line scan). Note the oscillation in the calcium-induced fluorescence. $D$, Same as $C$, but for the adjacent pixel (pixel 31 ). Error bars $( \pm 1$ SEM) were added, to illustrate that the oscillations are highly significant statistically. Laser power, $10.5 \mathrm{~mW}$; PMT, 803. Continuous perfusion with normal lizard Ringer's solution.

This results in a scanning visit to the same spot once every 20 msec. Figure $5 A$ shows the average fluorescence of 2000 conseculive line scans, passing through two synaptic boutons. All 64 pixels composing the line were analyzed. The 2000 sequential samples of one of the pixels are presented in Figure $5 B$. To determine whether there are oscillations in $[\mathrm{Ca}]_{\text {in }}$ at a single pixel, an autocorrelation was performed on these 2000 points. The results show clear oscillations, with a periodicity of $2.3 \mathrm{sec}$ (Fig. $5 C$ ). The oscillations were also clearly seen in the ncighboring pixels, one of which is illustrated in Figure $5 D$. The error bars show that the observed oscillations in the calcium signal were highly significant. 
Figure 6 shows that there was a significant positive correlation (at zero lag) between pixel 49 and the adjacent pixels. There was no measurable phase shift in the cross-correlations between different pixels within the same bouton (data not shown), indicating that the oscillations were almost synchronous at adjacent locations. In this experiment, there was a very strong correlation between the two boutons appearing in the line scan. This was not always the case; in the experiment shown in Figure 10 , where the line scanned three boutons, there was a significant correlation between the two boutons on the left, but they were not correlated with the bouton on the right.

Altogether, line scan experiments were performed on 37 synaptic boutons. Out of these 37 boutons, 19 were analyzed in detail.

In some experiments, the oscillations in fluorescence had an intermittent appearance. In one experiment, 16,000 line scans were performed along the one line; the scans were subdivided into eight groups of 2000 scans, and autocorrelation analysis was performed on each group scparatcly. Clear and significant oscillations were observed in the first, second, and fifth group, but not in the other groups, indicating that the oscillations were not persistent. Altogether, in about two-thirds of the boutons examined by the line scan (12 out of 19), the autocorrelation analysis revealed striking oscillations of fluorescence at many pixels, with a period of approximately $2.0 \mathrm{sec}$. In a number of experiments, the scanning line passed through more than one bouton at the same neuromuscular junction. Hence, both boutons were scanned almost simultaneously. Figure $7, A$ and $B$, shows such a simultaneous scanning. Although both boutons showed oscillations with a similar duration, the amplitude of the oscillations was much larger in the bouton illustrated in Figure $7 A$ compared to that illustrated in Figure $7 B$. Figure 7, $C$ and $D$, illustrates two additional examples from two different experiments. In Figure $7 D$ there was a substantial DC componcnt in the autocorrelation, which we attribute to the rather large bleaching encountered in this experiment.

The duration of the scanning imposes an upper limit on the period of the oscillation that can be detected. In four experiments, long scanning sequences were obtained (more than 100 sec). In these experiments, slower oscillations with a period of approximately $20 \mathrm{sec}$ were observed (Fig. $7 E, F$ ), in addition to the fast oscillations illustrated in Figure $7 A-D$. At some boutons, both types of oscillations could clearly be observed.

\section{Control experiments and variance analysis}

Optical imaging of biological preparations is a "noisy" process (cf. Foskett and Grinstein, 1990). In principle, there are at least five different groups of sources for the optical signal fluctuations: photon shot noise, instrumental noise (which includes laser output fluctuations, photomultiplier noise, electronic noise, and external interference noise), fluorophore fluctuations, artifactual biological fluctuations, and genuine biological fluctuations in the calcium signal. Therefore, before accepting the conclusion that the coordinated fluctuations and oscillations shown in Figures 2-7 represent a biological phenomenon, we must exclude the possibility that they are due to an instrumental, a methodological, or a biological artifact.

To assess the effect of photon shot noise and instrumental noise, we repeated the experiments and analyses using artificial fluorescent beads. Figure $8 A$ shows the average fluorescence map for part of a bead. Then one of the lines was selected and the correlation was estimated between each one of the pixels and all the pixels along the same line. One of these correlations is

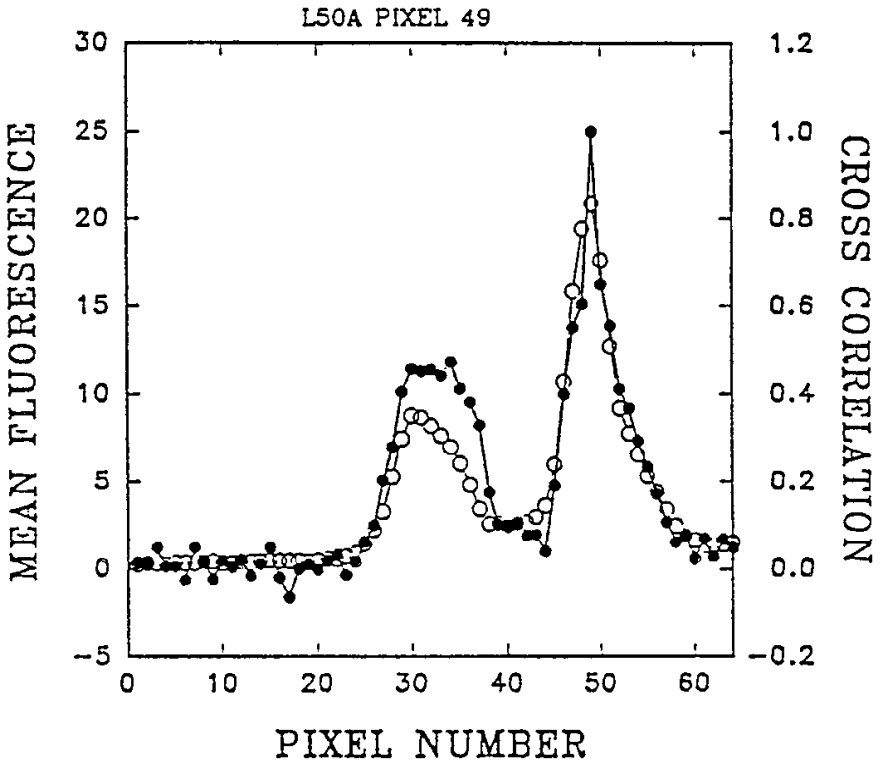

Figure 6. Cross-correlation between locations: same experiment as in Figure 5. Pixel 30 was used as a reference and the cross-correlations of all other pixels were estimated relative to it. Open circles, average fluorescence; solid circles, correlation coefficients between each pixel and pixel 49.

shown in Figure $8 B$. The only statistically significant correlation was the self correlation between the pixel and itself. All the other correlation coefficients are not significantly different from zero. The same treatment was applied to five additional beads and yielded similar results. Autocorrelation analysis revealed no significant oscillations in the bead's fluorescent signal (Fig. 8C). Analysis of fluorescence in pixels located in the muscle also showed no significant autocorrelation (data not shown). It appears, therefore, that the observed coordinated fluctuations and oscillations are not due to an instrumental noise.

Before proceeding to examine possible artifactual fluctuations, we would like to assess to what extent the observed fluctuations at synaptic boutons exceed those produced by the instrumentation. To obtain a quantitative measure of this excess, we performed a variance analysis of the signal.

Variance analysis (for dctails, sce Lloyd, 1980) was performed on artificial beads (Fig. 9). Figure $9 A$ shows the mean fluorescence from 2000 line scans. For each pixel, the variance was estimated (Fig. 9B) and plotted as a function of the mean fluorescence (Fig. 9C). In this and in six additional experiments with artificial beads, the variance was almost a linear function of the mean fluorescence under our experimental conditions. [It should be noted that if the laser power output is further increased, the variance-mean fluorescence relation has a progressively decreasing slope; it reaches a maximum and then decreases (V. Aviv, Y. Smith, and R. Rahamimoff, unpublished results).] To determine the desired working range, the coefficient of variation ( $\mathrm{SD} / \mathrm{mean})$ was plotted as a function of the mean fluorescence (Fig. 9D). At very low fluorescence signals, the large coefficient of variation imposes a lower limit on the biological experiments. Figure $9 E$ shows the distribution of the fluorescence signals originating from the artificial bead.

Figure 10 shows a similar analysis done using line scans performed at the lizard neuromuscular junction. Figure $10 \mathrm{~A}$ shows the fluorescence profile, where three synaptic boutons were clearly seen. The mean fluorescence in this experiment was in the same range as the artificial beads (Fig. 9). Figure $10 B$ shows the vari- 

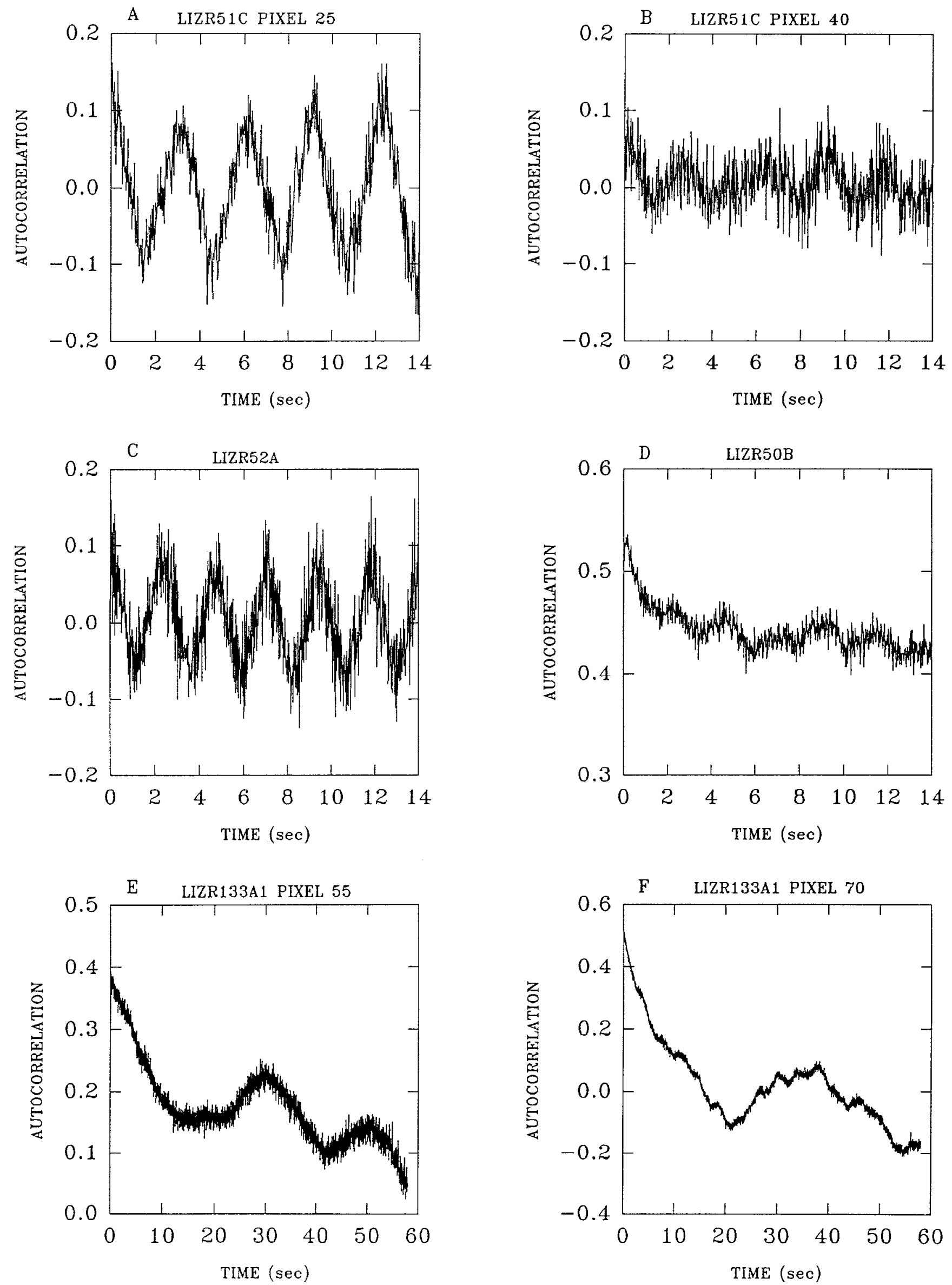

Figure 7. Six examples of fast and slow calcium oscillations in six different boutons, from four separate experiments. For details, see Results. 

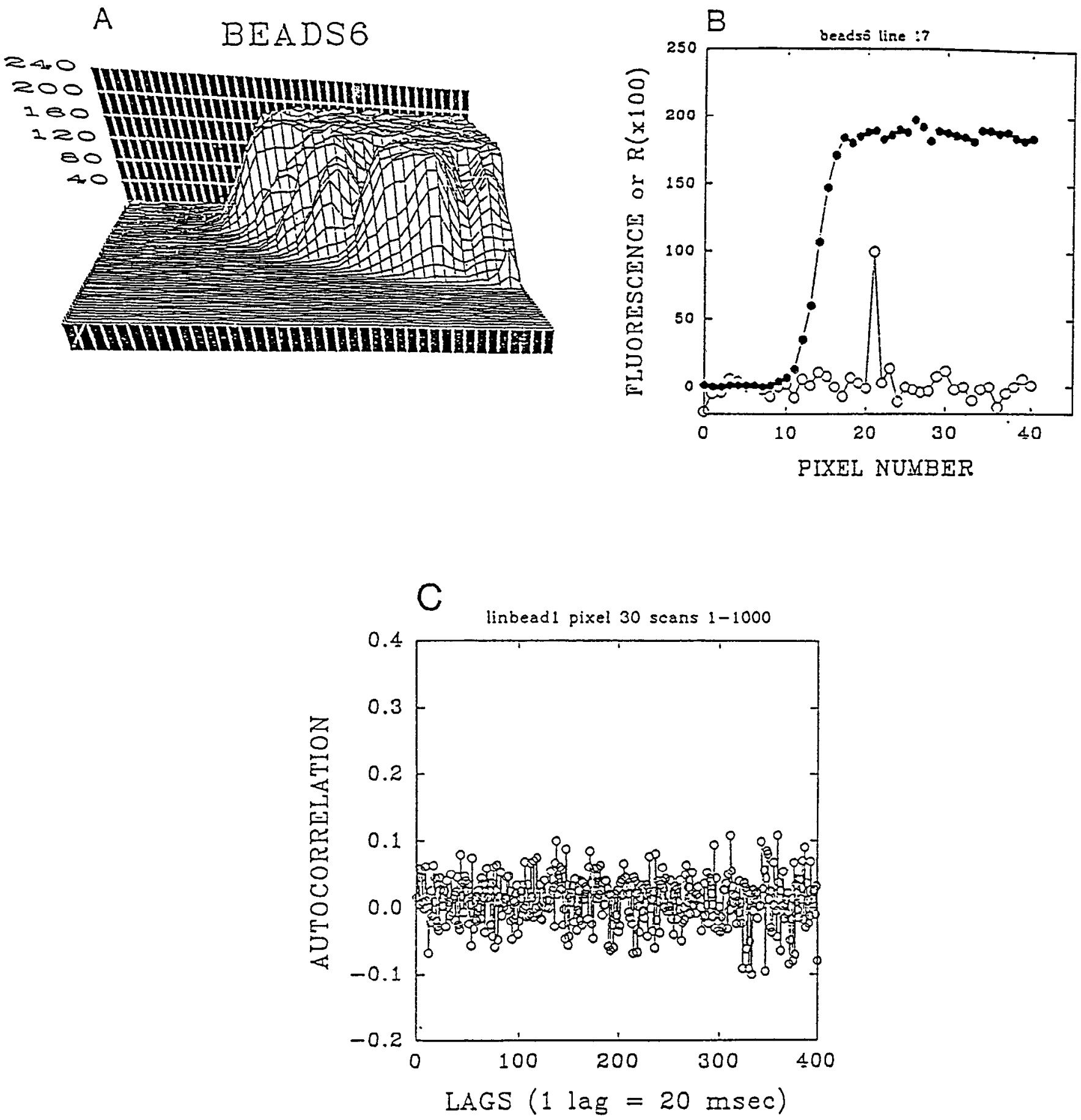

Figure 8. Fluorescence and corrclations of an artificial fluorescent bead. $A$, Three-dimensional map of the averaged fluorescence of a part of the bead. $B$, Same as Figure $4 A$, but for the artificial beads. All correlation coefficients were illustrated in reference to pixel 22 . Note the lack of any significant correlation. solid circles, average fluorescence; open circles, correlation coefficient $\times 100$. PMT, $975 ;$ laser power, $13 \mathrm{~mW}$; NDF, $10 \%$. Excitation at $514 \mathrm{~nm}$ and emission above $530 \mathrm{~nm}$ as in the experiments with the lizard neuromuscular junction. Image scan of $128 \times 128$. Pixel displacement of $0.5 \mu \mathrm{m}$. $C$, Lack of correlation in a fluorescent bead scanned with the line scan mode. PMT, 773; laser power, $10.4 \mathrm{~mW}$; NDF, $30 \%$. While $A$ and $B$ were done in the image scan mode, $C$ was done in the line scan mode.

ance profile of this neuromuscular junction. Plotting variance against mean fluorescence produced a picture (Fig. 10C) significantly different from that seen with artificial beads (Fig. $9 \mathrm{C}$ ). The initial part of the relation remained linear, but at the higher values of mean fluorescence that correspond to the synaptic boutons, the variance was much larger than that expected from the measurements on the artificial beads. Since variance caused by independent sources is additive (Lloyd, 1980), we attempt to separate between the instrumental and the biological variances. We assume that the initial linear part of the variancemean fluorescence relation (Fig. 10C) represents instrumental noise, since it comes from pixels in the background; we know from the bead experiments that instrumental variance is an almost linear function of the mean fluorescence; hence, we cal- 

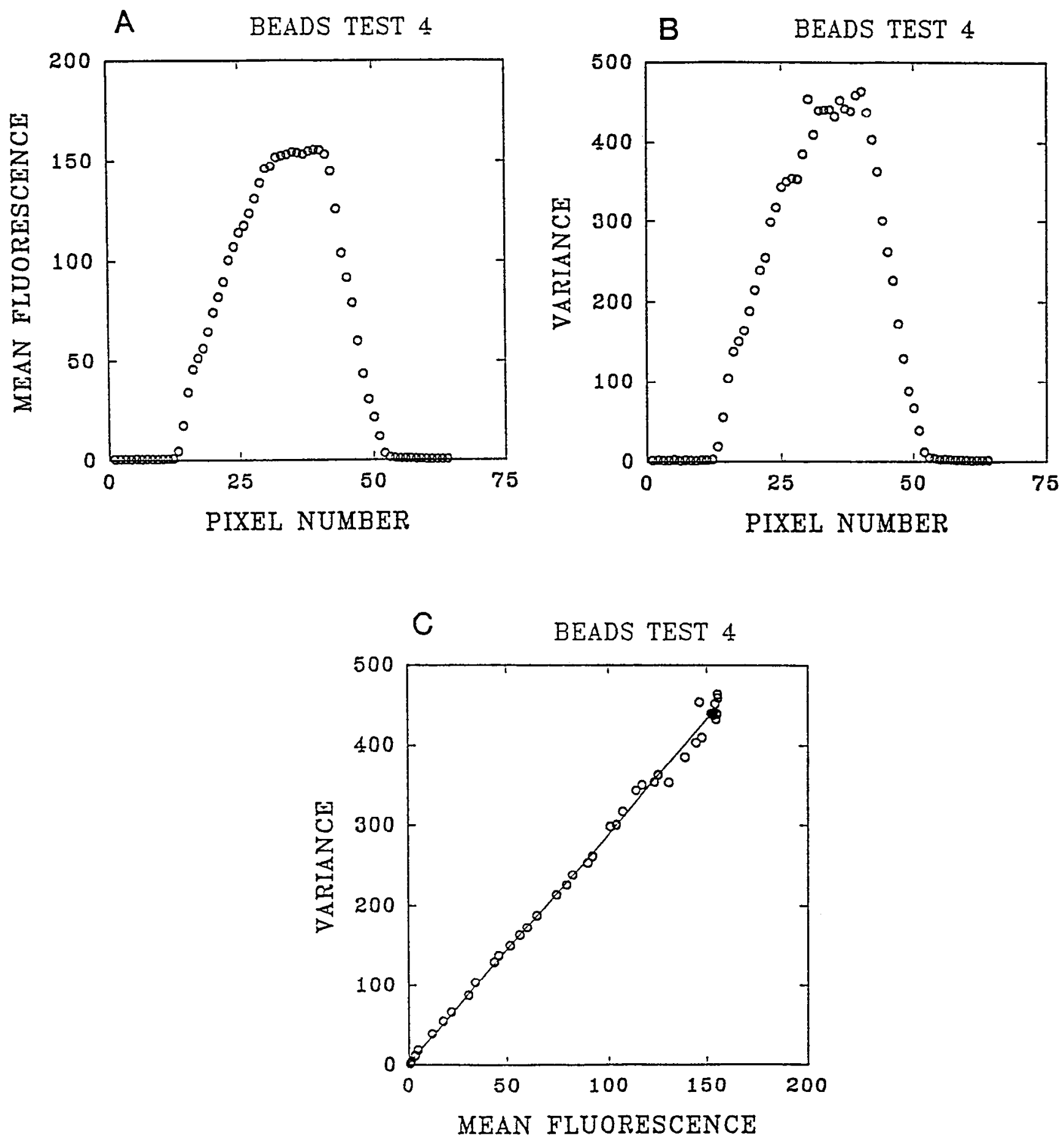

Figure 9. Variance analysis of fluorescent beads. $A$, The fluorescence profile of a bead (average of 2000 scans). $B$, The variance profile of the same bead. $C$, The variance against the mean fluorescence. Note that the data could be well represented by a straight line. The coefficient of correlation of the line $r=0.99$. D, The coefficient of variation as a function of the mean fluorescence. When the mean fluorescence is low, the coefficient of variation is high; hence, all of the biological experiments were done with mean fluorescence above 10. $E$, Histogram of the fluorescence signals of three pixels $(36,37,38)$ that have about the same mean fluorescence. Mean $=154.66$; SD $=21.09$. PMT, $800 ;$ laser power, $11.0 \mathrm{~mW}$. A 64 pixel line. Pixel displacement of $0.5 \mu \mathrm{m}$.

culated a linear regression on the initial part of the variancemean fluorescence relation (Fig. 10D) and extrapolated it to the higher values (Fig. 10D, solid circles). These extrapolated values were subtracted from the variance values to estimate the additional variance observed at the site of the synaptic boutons (Fig. 10E). Figure $10 F$ shows the same analysis in a slightly different way: on the ordinate the ratio between the total vari- ance and the instrumental variance is given as a function of the mean fluorescence. Figure $10 G$ demonstrates that this high variance ratio is found at the sites of the synaptic boutons. [For a discussion on the usage of the variance ratio, see Yule and Kendall (1965).] This again indicates that there is a very significant biological variance, in addition to the instrumental variance. The same conclusion could be reached also by a compar- 


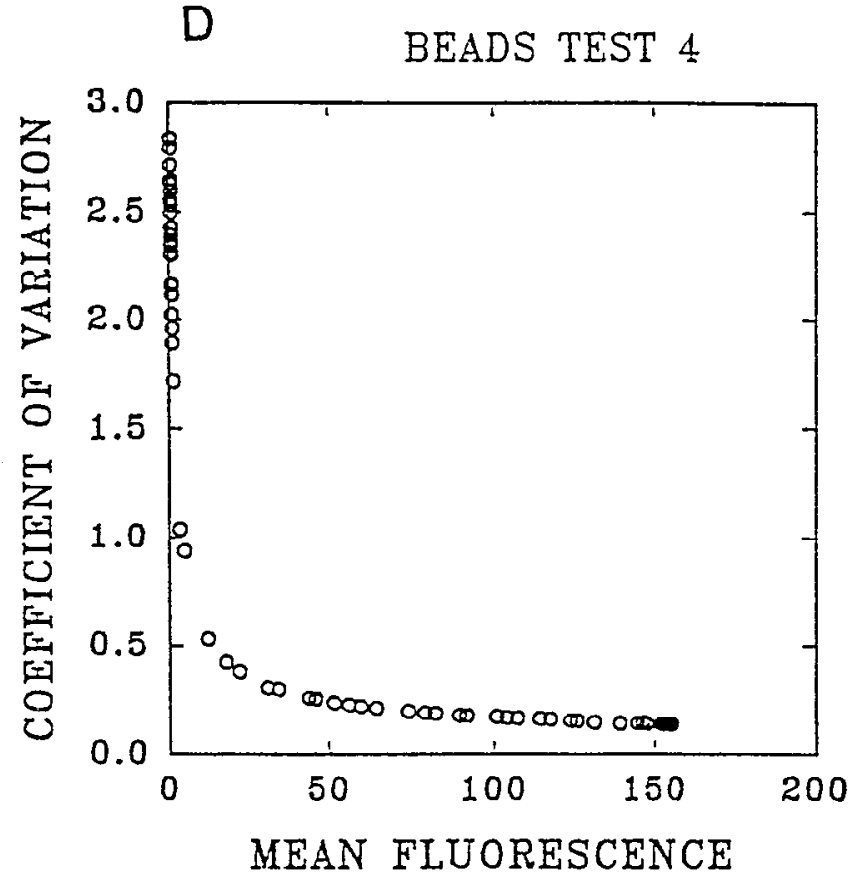

Figure 9. Continued.

ison of the means and the SDs of the fluorescence signals at artificial beads (Fig. 9) and of the synaptic boutons (Fig. 10H).

We assume that the extra variance observed in the area of the synaptic bouton is due to calcium fluctuations inside the nerve terminal. We therefore wanted to examine whether the extracellular calcium concentration $[\mathrm{Ca}]_{o}$ has an effect on the variance profile of the neuromuscular junction. When [Ca $]_{o}$ was reduced from $2.0 \mathrm{~mm}$ to $0.4 \mathrm{~mm}$, two changes were observed. First, the mean fluorescence at the site of the synaptic boutons was reduced. Second, there was a substantial reduction of the variance (Fig. 11 A). The results in Figure 11 are from the same experiment as these in Figure 10. Hence it is possible to compare the mean-variance relation and the variance ratio. The variance ratio in the low extracellular calcium medium (Fig. 11B) was much smaller at the sites of the synaptic boutons, compared to the control (Fig. 10G).

\section{Variance due to bleaching}

In addition to the instrumental and the biological sources for the variance, there is also a methodological source. Because the laser beam that excites the fluorophore also bleaches it, there was a continuous reduction in the fluorescence as the experiment progressed. In the standard calculation of variance, the differcnccs betwecn each individual value and the mean value are taken. But since there was a bleaching, the "mean value" was not constant. This introduced an extra "bleaching variance"" into the calculation. To overcome this problem, the bleaching was estimated by fitting an exponential regression function to the experimental results (Fig. 12A). The mean at every time point was calculated from the regression (Fig. 12B), and the variance was estimated from the "moving mean." In most pixels, this correction produced less than a $10 \%$ decrease in the total variance. In some boutons, however, the bleaching variance was responsible for about $50 \%$ of the total variance. Figure $12 C$ illustrates the correction of the variance due to bleaching in the experiment shown in Figure 10. We were surprised that the bleaching had a differential effect on the different boutons:

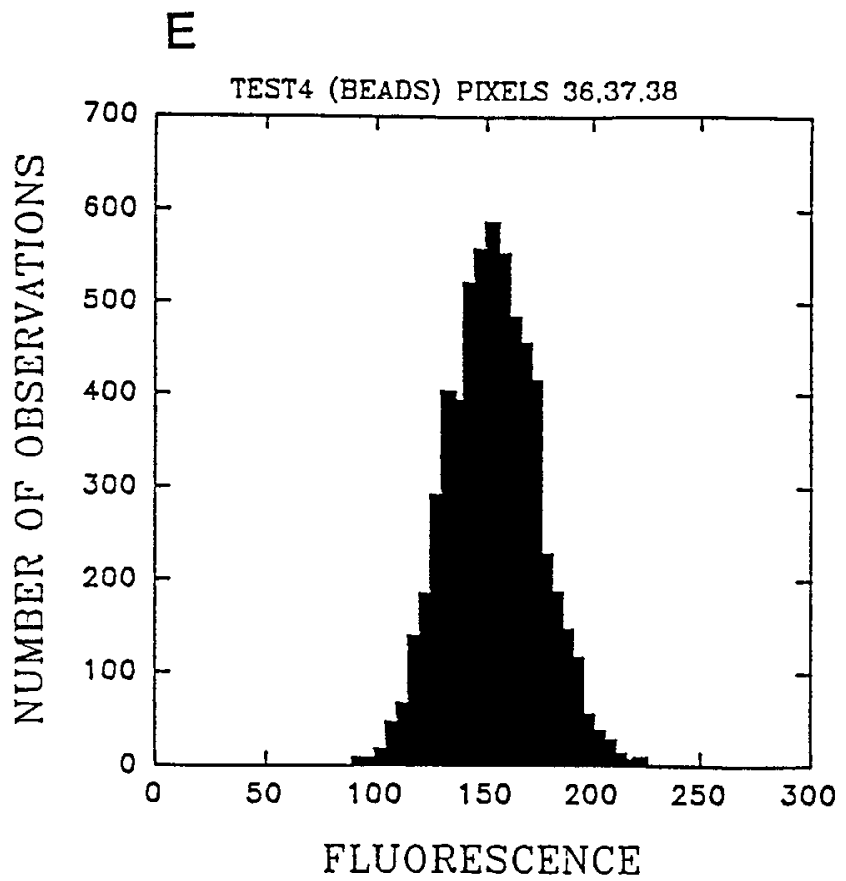

it had almost no effect on the variance in the middle bouton, but had a substantial effect on the right bouton (Fig. 12D). A plausible interpretation may be that the actual bleaching at locations exposed to the laser beam was much larger than what we observed, but in the line scan mode used in these experiments the bleaching was confined to only this line. Since the fluorophore molecules are mobile, there was a replenishment of these molecules from neighboring locations. Hence, the difference among boutons may represent the diffusion resistance of the fluorophore movement to the location of the line of scan.

\section{Fluorophore fluctuations}

Another plausible source for erroneous interpretation of our results is the possibility that the fluctuations occur not in the intracellular calcium concentration, but in the number of fluorophore molecules that are in the optical path. If there are waves in the concentration of the fluorescent dye, then these waves could be falsely interpreted as coordinated fluctuations in calcium concentration. To examine this possibility, we did the following analysis. If the fluorescent indicator molecules move from place to place and cause local inhomogeneities in their concentration, then an increase at one site should be accompanied by a decrease at another site. Figure 13 shows that this was not the casc. Figure $13 \mathrm{~A}$ shows the average fluorescence of 6000 line scans of a synaptic bouton. The 6000 scans were divided into 120 consecutive groups of 50 scans each (each group of 50 scans represents $1 \mathrm{sec}$ ). One of these groups is illustrated in Figure 13B. The difference between Figure $13 \mathrm{~A}$ and Figure $13 B$ is shown in Figure $13 C$, illustrating that all the pixels in the center of the bouton are above the average value. Dividing the differences by the mean fluorescence shows that in this set of scans the increase above the means was about $40 \%$ (Fig. 13D). The maximal amplitude of the fluctuation was $150 \%$ above the mean. Similar analysis on the image scans leads to the same conclusion, indicating that there is not a significant fluorophore movement at the confocal plane. Of course, an argument could be made that there are significant fluctuations 

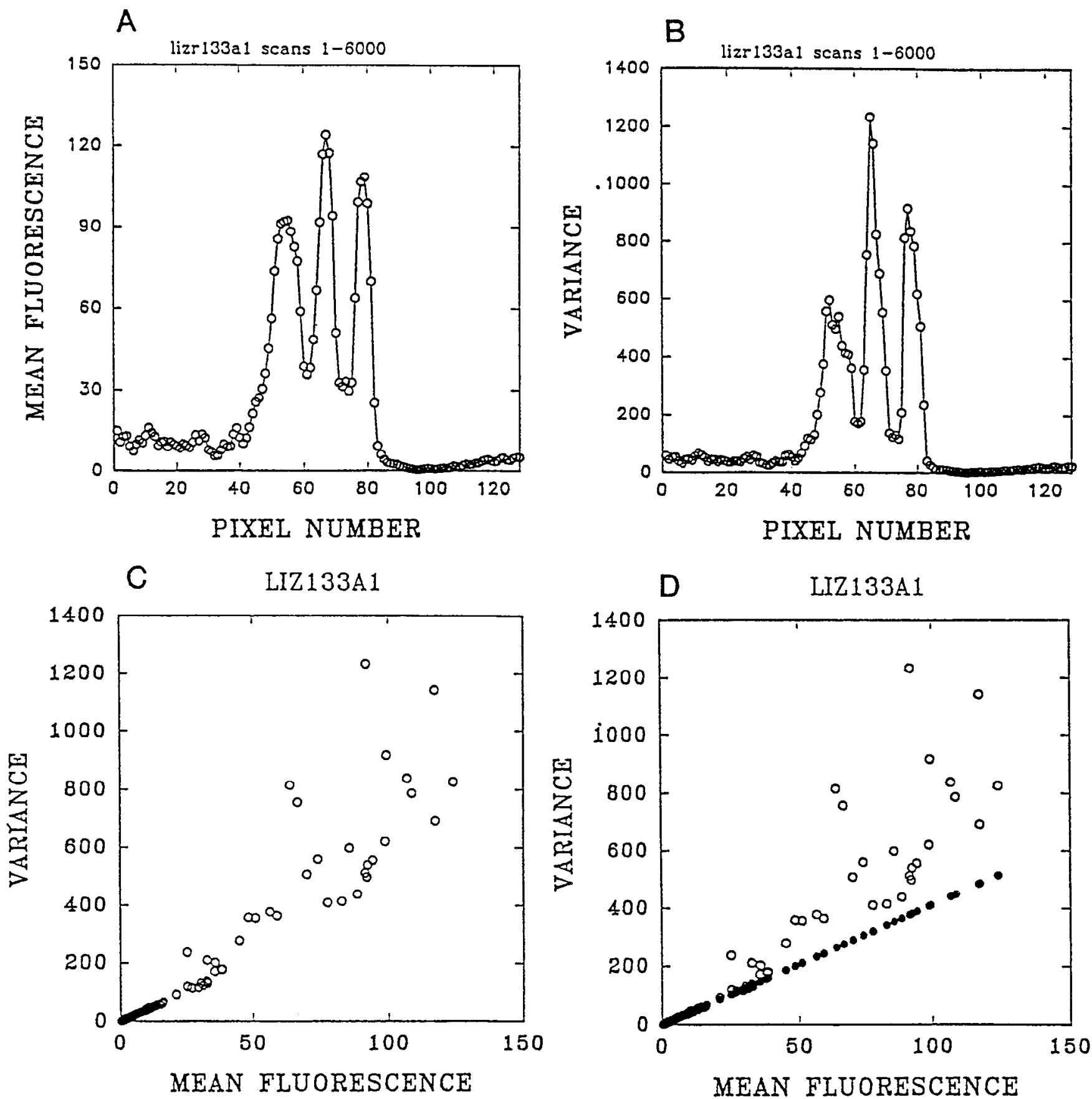

Figure 10. Variance analysis of boutons at the neuromuscular junction. $A$, A profile of three boutons at the neuromuscular junction (average of 6000 scans). Pixel displacement of $0.5 \mu \mathrm{m}$. $B$, The variance profile of the same boutons as in $A$. $C$, Variance against mean fluorescence of the synaptic bouton region. Note that the initial part of the graph (low mean fluorescence) has almost a linear relation with the variance. $D$, Same as $C$, but the initial linear part was extrapolated to the entire range (solid circles). $E$, The net variance: the difference between the open and the solid circles in $D$. $F$, Variance ratio as a function of the mean fluorescence. The observed variance was taken from $B$; the expected variance was taken from the linear extrapolation in $D . G$, The variance ratio in $F$ plotted against the pixel number. Note that the high variance ratio values correspond to the bouton locations in $A$. $H$, Histogram of the fluorescence signal coming from one pixel (66) inside a bouton. PMT, 820; laser power, 12.0 . Line scan of 128 pixels. Pixel displacement of $0.5 \mu \mathrm{m}$. Continuous perfusion with normal lizard Ringer's solution.

in the fluorophore concentrations, but these fluctuations are strictly in the direction that is orthogonal to the confocal plane. To check this argument, we performed the line scan imaging using different pinhole sizes and thus different widths of the confocal plane. Again we observed clear coordinated fluctuations in the optical signal. These experiments exclude the possibility that a major source for the fluctuation in the calcium signal is the movement of the fluorophore molecules inside the nerve terminals.

The type of analysis shown in Figure 13 was useful also in another aspect of this work; in some experiments a slight movement of the preparation occurred during the scanning process. Such a movement was easily detected in the consecutive average scans as a displacement of the boundary between the bouton 


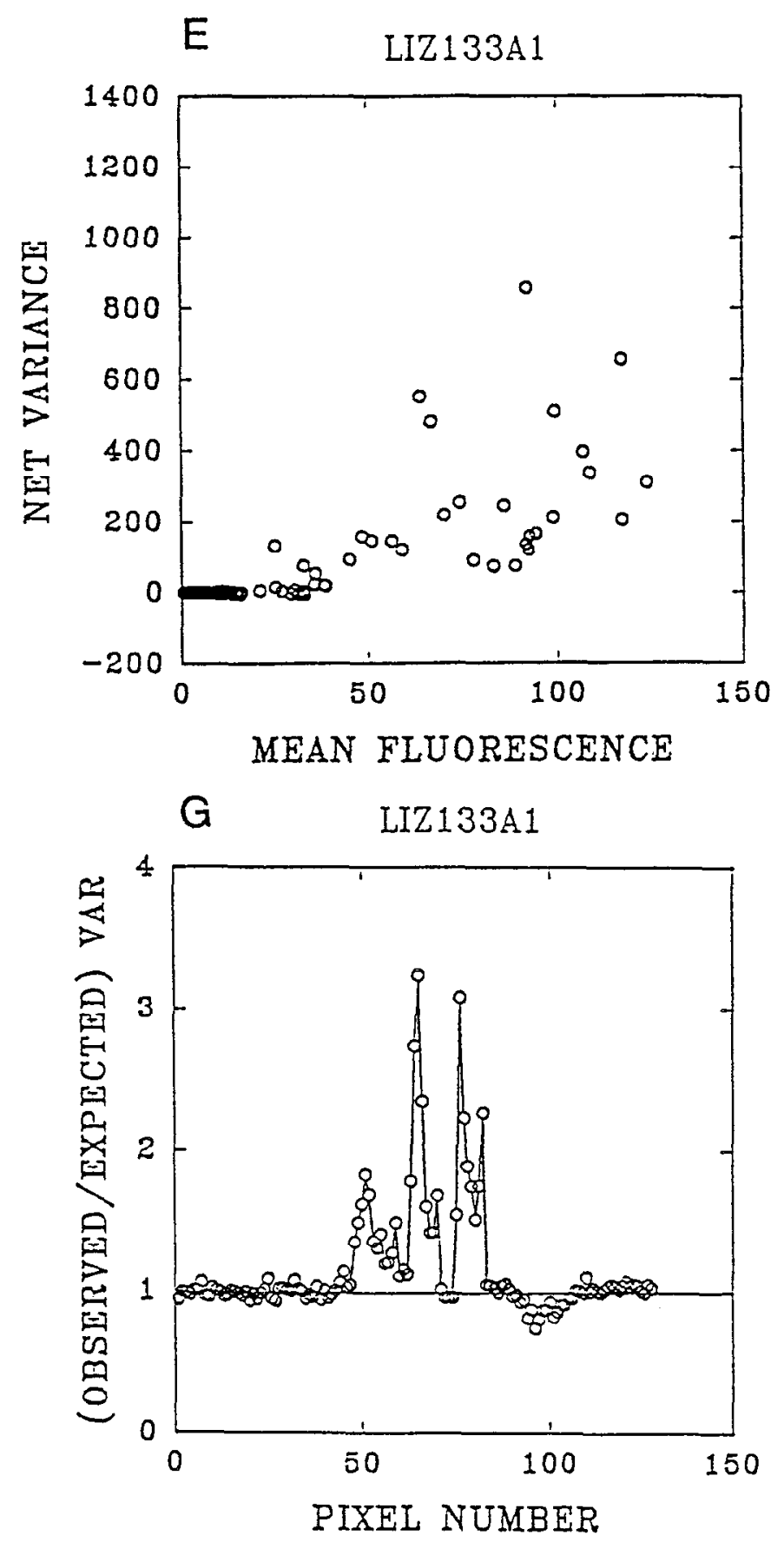

Figure 10. Continued.

and the surroundings. Experiments were discarded when a movement was detected. This type of analysis is useful to detect not only the movements in the horizontal plane, but also in the vertical plane. Before (and frequently also after) each sequence of line scans, a three-dimensional reconstruction of the bouton was performed. From this reconstruction, we know that the bouton does not have a regular shape; hence, a movement in the vertical direction should also change the location of the border between the bouton and the surroundings.

\section{Curare experiments}

The final control in this series of experiments was to examine whether the fluctuations and the oscillations could be observed
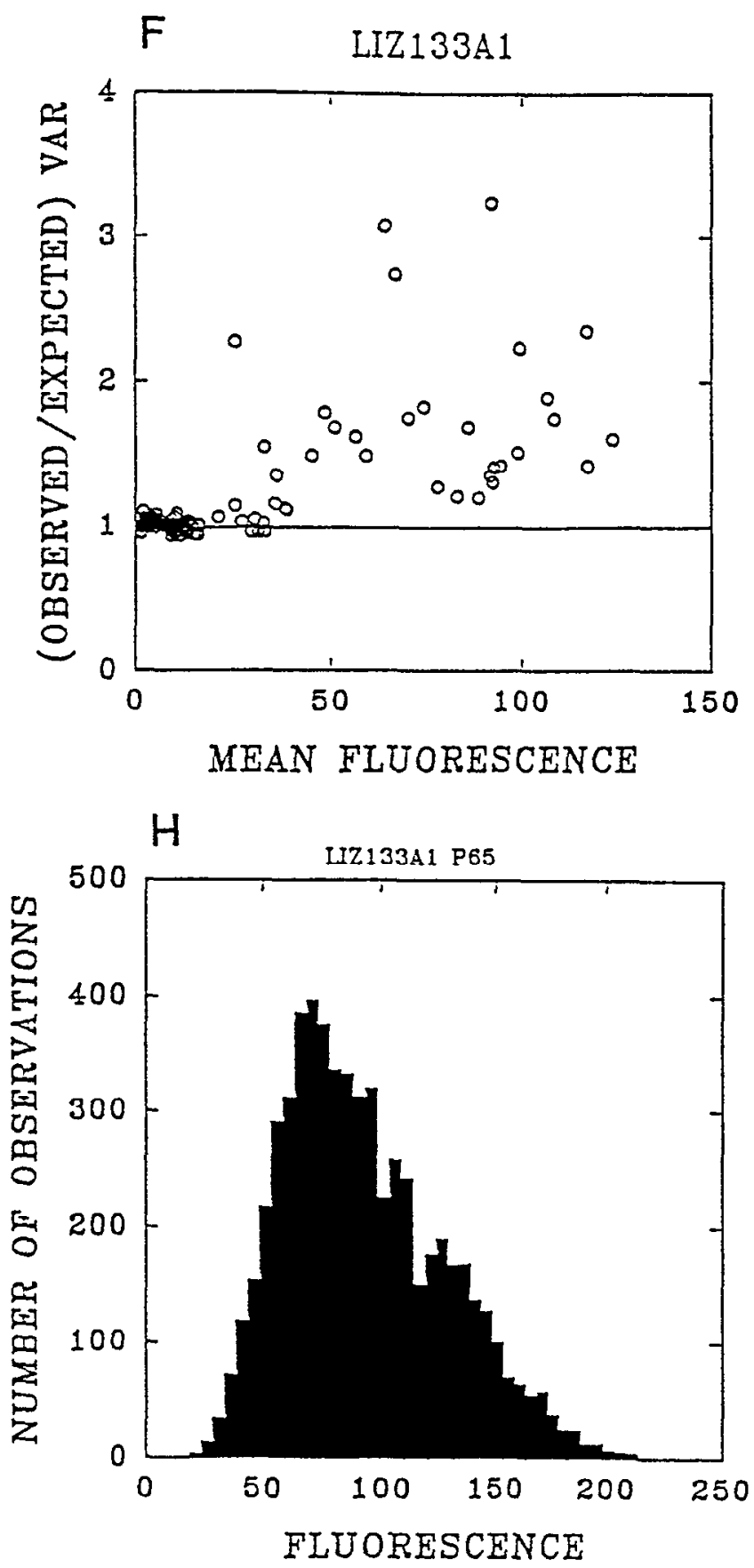

also when the muscle fiber was paralyzed by curare. Figure 14 shows that this indeed was the case. Clear oscillations in the calcium signal were observed also when enough curare was perfused through the medium to abolish the contraction of the muscle by nerve stimulation.

\section{Discussion}

In this work, we show that the long-wavelength calcium indicator Rhod-2 (Minta et al., 1989a,b) could be used to visualize the spontaneous changes in intracellular calcium in single synaptic boutons at the lizard neuromuscular junction. Our main concern was to distinguish between biological fluctuations and fluctuations originating from instrumental and methodological 

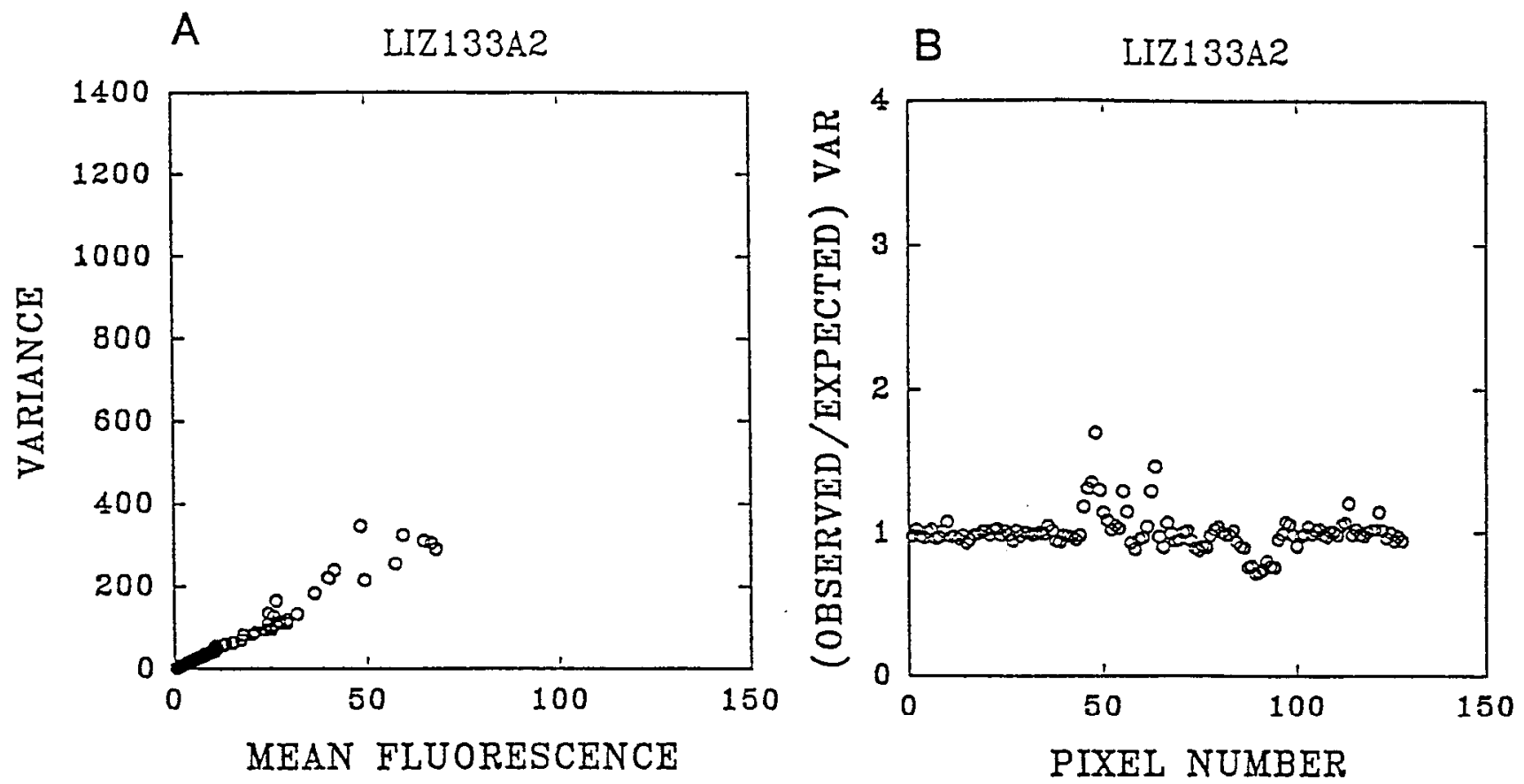

Figure 11. The effect of lowering extracellular calcium concentration on the fluorescence variance. $A$, Plot of variance against mean fluorescence of the same three boutons after 20 min perfusion in low Ca Ringer's $(0.4 \mathrm{~mm}$ calcium and $3.6 \mathrm{~mm} \mathrm{Mg})$. Note the decrease in mean fluorescence and in variance (compare with Fig. 10C). B, The variance ratio at low calcium. Same experimental conditions as in Figure 10, except the composition of the perfusion solution, which contained a lower $[\mathrm{Ca}]$ and a higher $[\mathrm{Mg}]$

sources. For this purpose, we scanned artificial beads; the optical signals from the artificial beads were in the same intensity range as the optical signals from the synaptic boutons. Both types of signals were subjected to the same statistical analysis, but produced strikingly different results. While in the synaptic boutons there are very clear coordinated fluctuations in the space domain and oscillations in the time domain, no such phenomena are observed in the artificial fluorescent beads. The results obtained with artificial beads served also to distinguish between the different sources of variance. It was found that the biological noise in the calcium signal is significantly larger than the instrumental one. Reduction in the extracellular calcium concentration greatly reduced this biological variance. We conclude, therefore, that the calcium concentration in the resting synaptic boutons undergoes systematic changes.

\section{Calcium fluctuations and oscillations}

The main finding in this article is that calcium fluctuations and oscillations were found in the critical part of the neuron responsible for synaptic transmitter release: the synaptic bouton. Previously, calcium oscillations were observed in glial cells (Cornell-Bell et al., 1990; Jensen and Chiu, 1990) and in the postsynaptic cells of the developing neocortex (Yuste and Katz, 1991); these oscillations were transmitter induced. Here we show that calcium oscillations could be detected in resting synaptic nerve terminals. Hence, the nervous system joins a growing number of nonexcitable cells where oscillations in intracellular calcium have been shown (Bcrridgc and Irvinc, 1989; Rink and Jacob, 1989; Berridge, 1990; Tsien and Tsien, 1990; Tsunoda, 1991). The cellular mechanisms responsible for calcium oscillations are not yet clearly established, and they are a subject of an intense debate. They may involve calcium-induced calcium release, calcium channels in the surface membrane, and the cascades of second messengers. It is not inconceivable that the general principles proposed for cellular signaling that involve calcium oscillations (Berridge and Gallione, 1988; Berridge et al., 1988; Meyer and Stryer, 1988; Rink and Jacob, 1989; Jacob, 1990; Petersen and Wakui, 1990; Dupont et al., 1991; Rooney et al., 1991) may be of importance also in the synaptic communication process in the nervous system. Such calcium oscillations may participate in the "time keeping" machinery of the nerve terminal.

\section{Calcium oscillations and transmitter release}

The main function of the presynaptic nerve terminal is to rclcasc quanta of transmitter. Since the beginning of the understanding of the transmitter release process, it was realized that this is a stochastic process (Fatt and Katz, 1952; del Castillo and Katz, 1954; Boyd and Martin, 1956). Later, it was found that under certain experimental conditions it is possible to demonstrate oscillations in the number of transmitter quanta liberated by the nerve impulse (Meiri and Rahamimorr, 1978; Pawson and Grinnel, 1989; Malinow, 1991). The calcium dependence of the transmitter release oscillations led to the proposal that these oscillations originate from oscillations in intracellular calcium (Meiri and Rahamimoff, 1978). Is it possible that the observed oscillations in $[\mathrm{Ca}]$ in the resting synaptic bouton, found in the present work, control directly the liberation process? It is too early to answer this question in the affirmative. At the frog neuromuscular junction, where oscillations in the evoked release wcre obscrved, no oscillations in spontaneous release were found when recordings were made from the entire neuromuscular synapse (Meiri, 1975). Also, no correlation was found between the spontaneously occurring miniature end-plate potentials and the nerve-evoked quantal liberation using intracellular recordings 

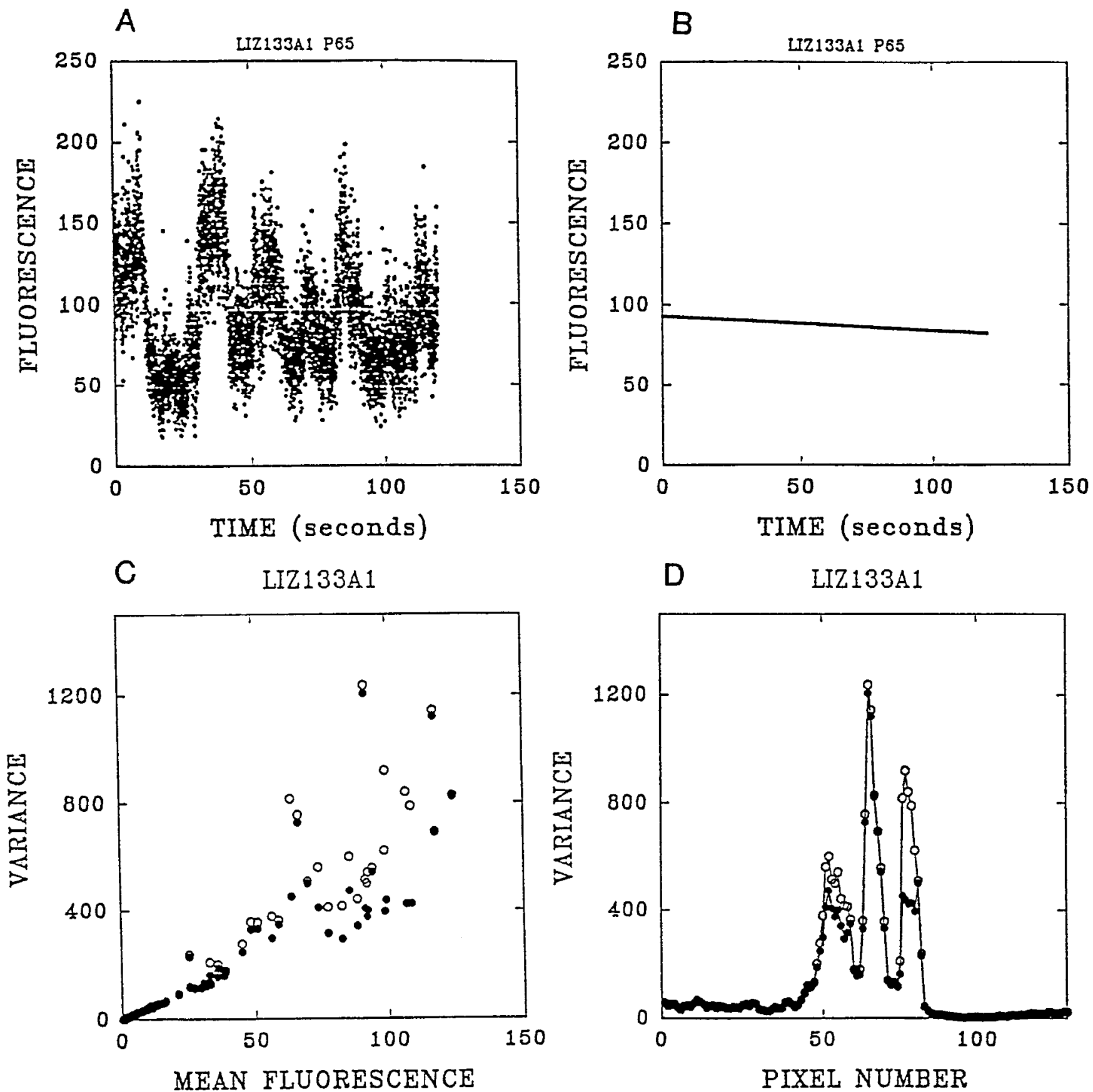

Figure 12. Estimation of the effect of fluorophore bleach on the variance. $A$, Raw data of 6000 scans in one pixel inside a bouton (same experiment as in Fig. 10). $B$, The data in $A$ were fitted with an exponential regression. The mean value was estimated from the regression, and the variance was estimated from this mean value. Since the bleaching was rather small, exponential decay does not appear very different from a linear decay. $C$, Variance estimates without (open circles) and with (solid circles) the correction for bleaching as a function of mean fluorescence. $D$, same as $C$, but a function of location. The data are from the same experiment illustrated in Figure $10 \mathrm{~A}$. Note that the correction for bleach was very small in the middle bouton, but was much larger in the right bouton.

from the entire synapse (Barrett et al., 1974). However, a significant correlation was found between the liberation of spontaneous and the nerve-evoked quanta when a recording was made extracellularly from a small portion of the nerve-muscle synapse (Barrett et al., 1974). This fits well with the observations made in this article, that the calcium signals in boutons are well correlated with those in some other boutons but definitely not with all the boutons at the same synapse.

It is tempting to view the calcium oscillations described here and the high power relation between calcium concentration and release (Dodge and Rahamimoff, 1967; Hubbard et al., 1968; Smith et al., 1985; for a review, see Augustine et al., 1987) as two facets of the same regulating system. However, in order to lend support to such a speculation, it will be necessary to examine whether the peak of the calcium signal precedes by a short time interval the actual liberation of the quantum. This will require, of course, the simultaneous recording of the optical and the electrical signals from the same synapse. 
Figure 13. Coordinated fluctuation in Rhod-2 fluorescence could not be explained by fluorophore fluctuations. $A$, Average fluorescence of 6000 scans on a synaptic bouton. $B$, The mean fluorescence of the same bouton as in $A$ in a period of $1 \mathrm{sec}(50$ scans). $C$, The difference between $A$ and $B$. Note that most of the bouton is above the average. $D$, The data in $C$ were normalized by the average fluorescence (in $A$ ), to estimate the percentage increase above the average.
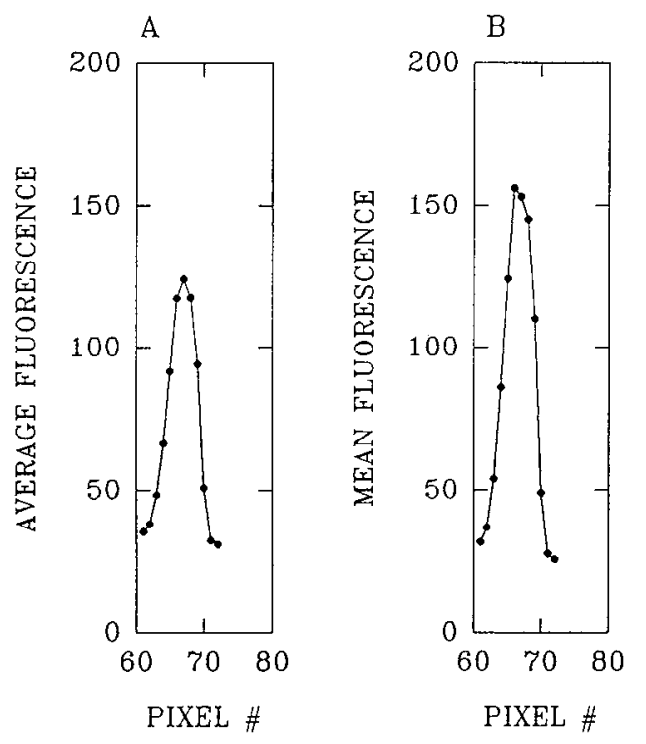
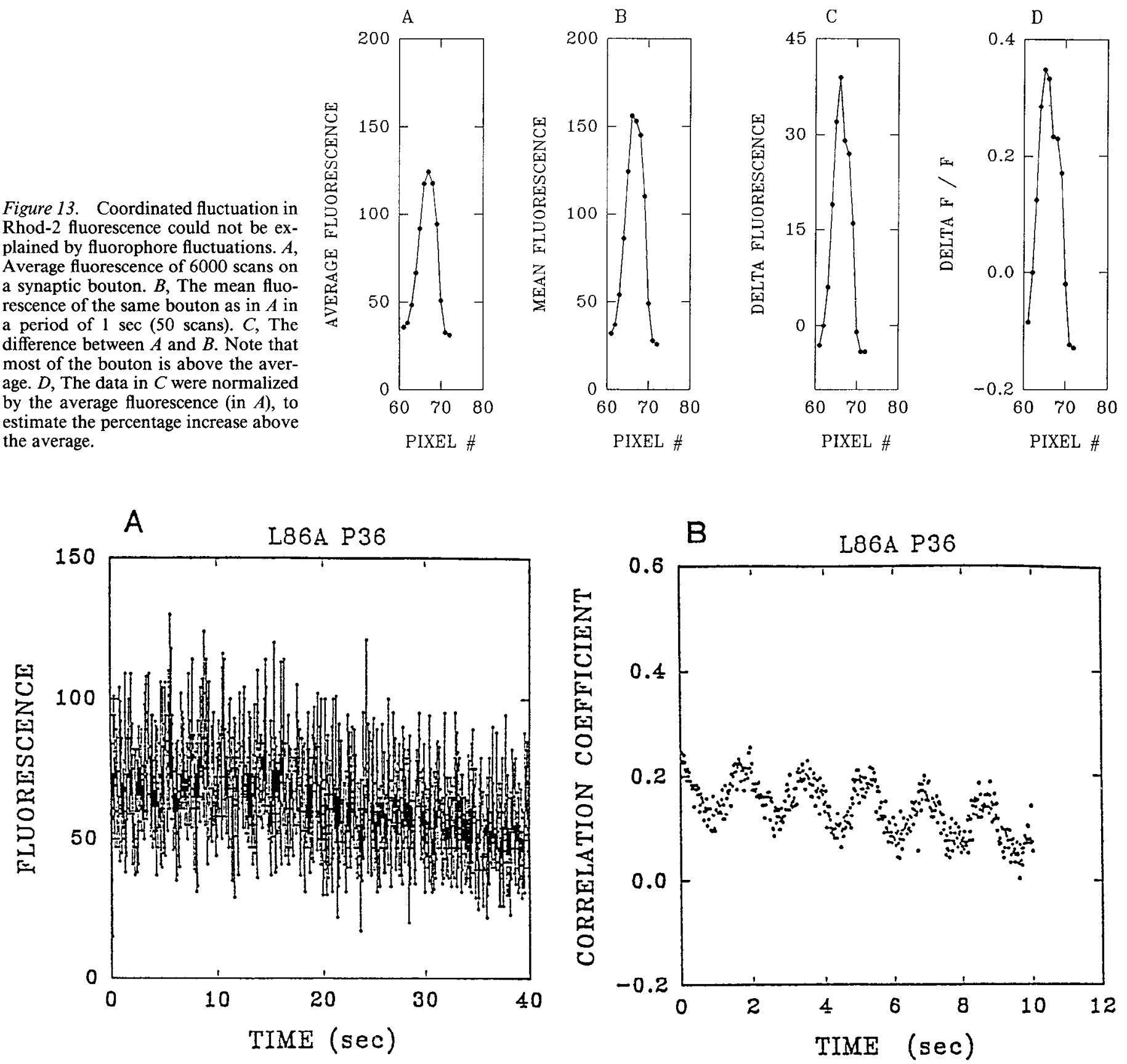

Figure 14. Oscillations in the fluorescence signals persist in the presence of a neuromuscular blocking agent: continuous perfusion of normal lizard Ringer's solution containing also $5.0 \mu \mathrm{M}$ curare. PMT, 820; laser power, $15.3 \mathrm{~mW}$. A line scan of 64 pixels. Pixel displacement was $0.5 \mu \mathrm{m}$.

\section{References}

Augustine GJ, Charlton MP, Smith SJ (1987) Calcium action in synaptic transmitter release. Annu Rev Neurosci 10:633-693.

Barrett EF, Barrett JN, Martin AR, Rahamimoff R (1974) A note on the interaction of spontaneous and evoked release at the frog neuromuscular junction. J Physiol (Lond) 237:453-463.

Barrett EF, Morita K, Scappaticci KA (1988) Effects of tetraethylammonium on the depolarizing after-potential and passive properties of lizard myelinated axons. J Physiol (Lond) 402:65-78.

Berridge MJ (1990) Calcium oscillations. J Biol Chem 265:95839586.

Berridge MJ, Galione A (1988) Cytosolic calcium oscillators. FASEB J 2:3074-3082.

Berridge MJ, Irvine RF (1989) Inositol phosphates and cell signalling. Nature 341:197-205.
Berridge MJ, Cobbold PH, Cuthbertson KSR (1988) Spatial and temporal aspects of cell signalling. Philos Trans R Soc Lond [Biol] 320: 325-34.3.

Boyd IA, Martin AR (1956) The end-plate potential in mammalian muscle. J Physiol (Lond) 132:74-91.

Carlsson K, Åslund N (1987) Confocal imaging for 3-D digital microscopy. Applied Optics 26:3232-3238.

Cornell-Bell AH, Finkbeiner SM, Cooper MS, Smith SJ (1990) Glutamates induces calcium waves in cultured astrocytes: long-range glial signaling. Science 247:470-473.

del Castillo J, Katz B (1954) Quantal components of the end plate potential. J Physiol (Lond) 124:560-573.

Dodge FA Jr, Rahamimoff R (1967) Co-operative action of calcium ions in transmitter release at the neuromuscular junction. J Physiol (Lond) 193:419-432. 
Dupont G, Berridge MJ, Goldbeter A (1991) Signal induced $\mathrm{Ca}^{2+}$ oscillations: properties of a model based on $\mathrm{Ca}^{2+}$ induced $\mathrm{Ca}^{2+}$ release. Cell Calcium 12:73-85.

Fatt P, Katz B (1952) Spontaneous subthreshold activity at motor nerve endings. J Physiol (Lond) 117:109-128.

Foskett JK, Grinstein S (1990) Noninvasive techniques in cell biology. New York: Wiley.

Grynkiewicz G, Poenie M, Tsien RY (1985) A new generation of $\mathrm{Ca}^{2+}$ indicators with greatly improved fluorescence properties. J Biol Chem 260:3440-3450.

Hubbard JI, Jones SF, Landau EM (1968) On the mechanism by which calcium and magnesium affect the release of transmitter nerve impulses. J Physiol (Lond) 196:75-86.

Jacob R (1990) Calcium oscillations in electrically non-excitable cells. Biochim Biophys Acta 1052:427-438.

Jensen AM, Chiu SY (1990) Fluorescence measurement of changes in intracellular calcium induced by excitatory amino acids in cultured cortical astrocytes. J Neurosci 10:1165-1175.

Katz B (1969) The Release of neural transmitter substances, Vol 10 (The Sherrington lecture). Liverpool: I iverpool USP.

Lichtman JW, Sunderland WJ, Wilkinson RS (1989) High resolution imaging of synaptic structure with a simple confocal microscope. New Biologist 1:75-82.

Lindgren CA, Moore JW (1989) Identification of ionic currents at presynaptic nerve endings of the lizard. J Physiol (Lond) 414:201222.

Lindgren CA, Moore JW (1991) Calcium current in motor nerve endings of the lizard. Ann NY Acad Sci 635:58-69.

Lindgren CA, Moore JW, Sostman AH (1988) Pharmacological characterization of calcium channels in motor nerve terminals of the lizard. J Gen Physiol 92:3a.

Lloyd E (1980) Handbook of applicable mathematics, Vol II, Probability (Ledermann W, ed). Chichester: Wiley.

Malinow R (1991) Transmission between pairs of hippocampal slice neurons: quantal levels, oscillations and LTP. Science 252:722-724.

Meiri H (1975) An analysis of the probability properties of transmitter release at the neuromuscular synapse. $\mathrm{PhD}$ thesis, Hebrew University of Jerusalem.

Meiri H, Rahamimoff R (1978) Clumping and oscillations in evoked transmitter release at the frog neuromuscular junction. J Physiol (Lond) 278:513-523.

Melamed N, Rahamimoff R (1991) Confocal microscopy of the lizard motor nerve terminals. J Basic Clin Physiol Pharmacol 2:63-86.

Melamed N, Helm PJ, Rahamimoff R (1992) Confocal microscopy of the isolated lizard neuromuscular junction: calcium oscillations in resting synaptic boutons. J Physiol 452:47P.

Meyer T, Stryer L (1988) Molecular model for receptor-stimulated calcium spiking. Proc Natl Acad Sci USA 85:5051-5055.

Minta A, Gryzkiewicz, Tsien RY (1989a) Fluorescent probes of cell signaling. Annu Rev Neurosci 12:227-253.
Minta A, Kao JPY, Tsien RY (1989b) Fluorescence indicators for cytosolic calcium based on rhodamine and fluorescein chromophores. J Biol Chem 264:8171-8178.

Morita K, Barrett EF (1989) Calcium dependent depolarization originating in lizard motor nerve terminals. J Neurosci 9:3359-3369.

Morita K, Barrett EF (1990) Evidence for two calcium-dependent potassium conductances in lizard motor nerve terminals. J Neurosci 10:2614-2625.

Pawley JB (1990) Handbook of biological confocal microscopy. New York: Plenum.

Pawson PA, Grinnel AD (1989) Oscillation period of MEPP frequency at neuromuscular junction is inversely correlated with release efficacy and independent of acute $\mathrm{Ca}^{2+}$ loading. Proc R Soc Lond [Biol] 237: 489-499.

Petersen OH, Wakui M (1990) Oscillating intracellular $\mathrm{Ca}^{2+}$ evoked by activation of receptors linked to inositol lipid hydrolysis: mechanism of generation. J Membr Biol 118:93-105.

Proske U, Vaughan P (1968) Histological and electrophysiological investigation of lizard skeletal muscle. J Physiol (Lond) 199:495-509.

Rink TJ, Jacob R (1989) Calcium oscillations in nonexcitable cells. Trends Neurosci 12:43-46.

Robitaille R, Adler EM, Charlton MP (1990) Strategic location of calcium channels at transmitter release sites of frog neuromuscular synapses. Neuron 5:773-779.

Rooney TA, Renard DC, Sass EJ, Thomas AP (1991) Oscillatory cytosolic calcium waves independent of stimulatcd inositol 1,4,5 triphosphate formation in hepatocytes. J Biol Chem 266:12272-12282.

Sheppard CJR, Wilson T (1978) Depth of field in the scanning microscope. Opt Lett 3:115.

Smith SJ, Augustine GJ, Charlton MP (1985) Transmission at voltageclamped giant synapse of the squid: evidence for cooperativity of presynaptic calcium action. Proc Natl Acad Sci USA 82:622-625.

Tsien RW, Tsien RY (1990) Calcium channels, stores and oscillations. Annu Rev Cell Biol 6:715-760.

Tsunoda $\mathrm{Y}$ (1991) Oscillatory $\mathrm{Ca}^{2+}$ signaling and its cellular function. New Biologist 3:3-17.

Walrond JP, Reese TS (1985) Structure of axon terminals and active zones of synapses on lizard twitch and tonic muscle fibers. J Neurosci 5:1118-1131.

White JG, Amos WB, Fordham M (1987) An cvaluation of confocal versus conventional imaging of biological structures by fluorescence light microscopy. J Cell Biol 105:41-48.

Willard WA (1915) The cranial nerves of Anolis carolinensis. Bull Harv Univ Mus Comp Zool 2:17-135.

Yule GU, Kendall MG (1965) An introduction to the theory of statistics, 14th ed. London: Griffin.

Yuste R, Katz LC (1991) Control of postsynaptic $\mathrm{Ca}^{2+}$ influx in developing neocortex by excitatory and inhibitory neurotransmitters. Neuron 6:333-344. 